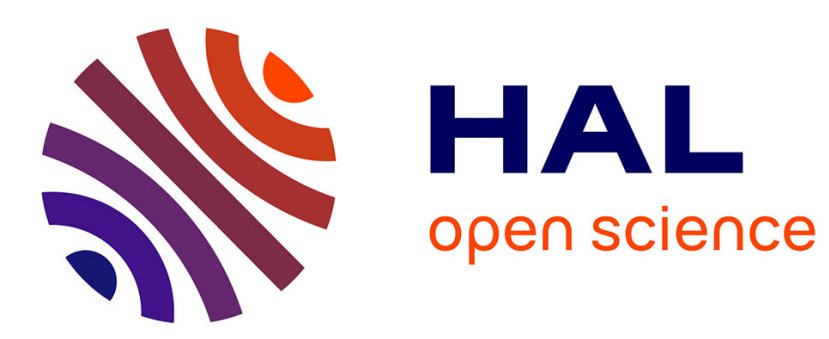

\title{
BIE and material differentiation applied to the formulation of obstacle inverse problems.
}

Marc Bonnet

\section{To cite this version:}

Marc Bonnet. BIE and material differentiation applied to the formulation of obstacle inverse problems.. Engineering Analysis with Boundary Elements, 1995, 15, pp.121-136. 10.1016/0955-7997(95)00011C . hal-00092369

\section{HAL Id: hal-00092369 \\ https://hal.science/hal-00092369}

Submitted on 9 Aug 2008

HAL is a multi-disciplinary open access archive for the deposit and dissemination of scientific research documents, whether they are published or not. The documents may come from teaching and research institutions in France or abroad, or from public or private research centers.
L'archive ouverte pluridisciplinaire HAL, est destinée au dépôt et à la diffusion de documents scientifiques de niveau recherche, publiés ou non, émanant des établissements d'enseignement et de recherche français ou étrangers, des laboratoires publics ou privés. 


\title{
BIE and material differentiation applied to the formulation of obstacle inverse problems *
}

\author{
Marc BonneT \\ Laboratoire de Mécanique des Solides \\ (URA CNRS 317, centre commun Polytechnique - Mines - Ponts et Chaussées) \\ Ecole Polytechnique, 91128 Palaiseau Cedex, France \\ bonnet@@athena.polytechnique.fr, (1)-69333026 (fax)
}

November 1994

\begin{abstract}
In this paper, we consider the problem of identifying, by means of boundary element methods and nonlinear optimization, a cavity or obstacle of unknown location and shape embedded in a linearly acoustic or elastic medium. The unknown shape is classically sought as to achieve a best fit between the measured and computed values of some physical quantity, which is here the scattered acoustic pressure field. One is usually led to the minimization of a cost function $\mathcal{J}$. Classical nonlinear optimization algorithms need the repeated computation of the gradient of the cost function with respect to the design variables as well as the cost function itself. The present paper emphasizes the formulation and effectiveness of the adjoint problem method for the gradient evaluation. First the hard obstacle inverse problem for $3 \mathrm{D}$ acoustics is considered. For a given $\mathcal{J}$, the adjoint problem is established, and the gradient of $\mathcal{J}$ is then formulated in terms of both primary and adjoint states. Next, the adjoint variable approach is extended to the case of a penetrable obstacle in a 3D acoustical medium, and also for a traction-free cavity in a $3 \mathrm{D}$ elastic medium. Explicit formulas for the gradient of $\mathcal{J}$ with respect to shape variations, which appear to be rather compact and elegant, are established for each case. The formulation is incorporated in an unconstrained minimization algorithm, in order to solve numerically the inverse problem. Numerical results are presented for the search of a rigid bounded obstacle embedded in an infinite 3D acoustic medium, where the measurements are taken to be values of the pressure field on a remote measurement surface, the obstacle being illuminated by monochromatic plane waves. They demonstrate the efficiency of the proposed method. Some computational issues (accuracy, CPU time, influence of measurements errors) are discussed. Finally, for the sake of completeness, the direct differentiation approach is also treated and new derivative BIE formulations established.
\end{abstract}

KEYWORDS: Inverse problem in scattering, Direct differentiation method, Adjoint variable approach, Domain derivative.

*Eng. Anal. with Boundary El., 15, 121-136 (1995) 


\section{Introduction}

The consideration of variations of integral functionals with respect to a geometrical domain arises in the study of many situations where a geometrical domain plays a major role. The present paper is focused on some geometrical inverse problems, where part of the domain boundary is unknown (e.g. in defect or crack identification). Its determination is usually attempted by minimizing a cost function $\mathcal{J}$ between computed (for a given domain configuration) and known (for the actual, unknown, domain configuration) values of some physical quantity.

Most usual optimization algorithms, such as conjugate gradient or BFGS variable metric methods, use first-order derivatives. Besides, it is a known fact that finite-difference estimations of gradients (which would require here finite perturbations of the geometrical domain), apart from being computationally expensive, constitute an ill-posed mathematical problem (Tikhonov and Arsenin [24]) and then are prone to inaccuracy. Hence one should seriously consider reverting to analytical differentiation with respect to a variable domain. This concept has been studied and used by many authors (see e.g. Haug et al. [15], Petryk and Mroz [22]), up to now mainly in FEM-oriented contexts. However, since in such problems the domain (and hence its boundary) is a primary unknown, it is a natural idea to consider boundary integral formulations, which offer in this context the "minimal" modelling.

The formulation of shape sensitivities, in a BIE context or otherwise, may result from either the adjoint problem method or the direct differentiation approach, applied either before and after discretization of the primary BIE. The adjoint problem approach to shape sensitivity in a BIE context has been considered e.g. by Aithal and Saigal [1], Choi and Kwak [11], Burczinski [10], Meric [19] or in [4]. The direct differentiation approach is treated, among others, in Barone and Yang [2] for strongly singular elastic BIE formulations and in [6] for regularized elastic BIE and second-order derivatives; see also Zhang and Mukherjee [25].

The present paper deals with the application of BIE and domain differentiation to some obstacle inverse problems, with emphasis on the use of the adjoint variable method. Given an incident wave and values of the scattered wave on some measurement surface, one seeks to reconstruct an unknown obstacle with given boundary conditions (rigid or penetrable obstacle in an acoustical medium or traction-free cavity in an elastic medium). The surrounding medium is here assumed to be infinite but this assumption is used for ease of exposition and is by no means essential. This kind of inverse problem has received attention in the literature, from both the mathematical perspective (Colton and Kress [13], Colton [12], Kress [17], among others) and the computational standpoint (see e.g. Nishimura and Kobayashi [20], [21]).

For this particular kind of problem, where a single functional is to be minimized, the adjoint variable approach seems to be more efficient than the direct differentiation method, for one adjoint problem has to be solved instead of $D$ sensitivity problems, $D$ being the number of parameters that describe geometry changes. Then, in the present paper, we emphasize the formulation and effectiveness of the adjoint problem approach in connexion with the minimization of the cost function $\mathcal{J}$. First the hard obstacle inverse problem for 3D acoustics is considered. For a given $\mathcal{J}$, the adjoint problem is established, and the gradient of $\mathcal{J}$ is then formulated in terms of both primary and adjoint states. Then, numerical examples using this formulation 
in conjunction with collocation BEM are presented for this problem. Next, the adjoint variable approach is extended to the case of a penetrable obstacle in a 3D acoustical medium, and also for a traction-free cavity in a 3D elastic medium. Explicit expressions for the gradient of $\mathcal{J}$ with respect to shape variations are given in each case. Finally, for the sake of completeness, the direct differentiation approach is also treated for Neumann boundary conditions on the obstacle.

\section{Inverse problem for a hard obstacle}

The identification of a 3D bounded rigid body $\Omega^{-}$, of regular boundary $\Gamma$, imbedded in an infinite acoustical medium $\Omega=\mathbb{R}^{3}-\Omega^{-}$, is considered. The cavity is subjected to a known harmonic incident pressure $p^{I}(\boldsymbol{y}) \exp (-i \omega t)$, which satisfies Helmholtz' equation $\left(\Delta+k^{2}\right) p^{I}=0$ in $\mathbb{R}^{3}$ ( $k$ : wavenumber); following the usual convention, the time-harmonic factor $\exp (-i \omega t)$ is implicit everywhere in the sequel.

Direct problem. In the presence of a known obstacle $\Omega^{-}$, the total pressure is $p^{T}=p^{I}+p$, where the scattered pressure $p$ solves the following direct problem, also called primary problem:

$$
\begin{cases}\left(\Delta+k^{2}\right) p=0 & \text { in } \Omega \\ p_{, n}+p_{, n}^{I}=0 & \text { on } \Gamma \\ \text { (radiation condition) } & \end{cases}
$$

where the unit normal $\boldsymbol{n}$ is directed outside $\Omega$, i.e. is interior to $\Gamma$. The solution $p$ to (1) depends on $\Gamma$; it is denoted $p_{\Gamma}$ to emphasize this fact.

Inverse problem. The unknown shape of $\Omega^{-}$, ie the surface $\Gamma$, is to be identified. Supplementary data is necessary in order to compensate for the unknown geometry in the direct problem (1). For example $p$ may take known values $\hat{p}$ on a measurement surface $C$ exterior to $\Gamma$. The unknown $\Gamma$ is thus searched so as to minimize a distance between computed $\left(p_{\Gamma}\right)$ and known $(\hat{p})$ values of $p$ on $C$ :

$$
\mathcal{J}(\Gamma)=J\left(p_{\Gamma}\right) \quad \text { with } \quad J(p)=\int_{C} j(p-\hat{p}) \mathrm{d} S
$$

For example, $2 j=|p-\hat{p}|^{2}$ gives the usual least-squares distance. Since the measurement surface $C$ is fixed, $\mathcal{J}$ depends on $\Gamma$ through $p_{\Gamma}$ only, while in other situations (e.g. in shape optimization), the geometrical support of the integral itself may be part of the unknown boundary.

The minimization of $\mathcal{J}(\Gamma)$ is best solved, in terms of both computational efficiency and accuracy, using gradient methods, such as Quasi-Newton or conjugate gradient (see e.g Fletcher [14]). These algorithms need repeated computations of the derivative of $\mathcal{J}$ with respect to (the design parameters which define the current location of) $\Gamma$. Using finite-difference evaluations is computationally expensive, because the evaluation of each partial derivative needs a complete solution of (1) on a perturbed geometry $(\Gamma+\delta \Gamma)$ and may be poor in terms of accuracy. An alternative possibility, which is the main topic of this paper, is the use of the analytical material differentiation concept, in either the adjoint problem approach (APA) or the direct differentiation approach (DDA) forms. 
Governing acoustic BIE. The boundary $\Gamma$ is itself the primary unknown of the inverse problem under consideration, while information at interior points is of no particular use, except of course on the measurement surface $C$. Then boundary integral equations (BIE) suggest themselves as the most appropriate discretization tool.

Recall that any solution $p$ to the inhomogeneous Helmholtz' equation $\left(\Delta+k^{2}\right) p=F$ on the exterior domain $\Omega$ with proper radiation conditions at infinity satisfies the following regularized BIE [3]:

$$
\begin{aligned}
& p(\boldsymbol{x})+\int_{\Gamma} p(\boldsymbol{y})\left[G_{, n}(\boldsymbol{x}, \boldsymbol{y})-G_{, n}^{0}(\boldsymbol{x}, \boldsymbol{y})\right] \mathrm{d} S_{y} \\
& \quad+\int_{\Gamma}[p(\boldsymbol{y})-p(\boldsymbol{x})] G_{, n}^{0}(\boldsymbol{x}, \boldsymbol{y}) \mathrm{d} S_{y}-\int_{\Gamma} q(\boldsymbol{y}) G(\boldsymbol{x}, \boldsymbol{y}) \mathrm{d} S_{y}+\int_{\Omega} F(\boldsymbol{y}) G(\boldsymbol{x}, \boldsymbol{y}) \mathrm{d} V_{y}=0
\end{aligned}
$$

in which $G(\boldsymbol{x}, \boldsymbol{y})=e^{i k r} /(4 \pi r)$ and $G^{0}(\boldsymbol{x}, \boldsymbol{y})=1 /(4 \pi r)$ are the dynamic and static fundamental solutions, while $r=|x-y|,(\cdot)_{n} \equiv n_{s}(\cdot)_{, s}, q \equiv p_{, n}$; the comma denotes partial derivatives with respect to the field point $\boldsymbol{y}:(\cdot)_{j} \equiv \partial(\cdot) / \partial y_{j}$. Equation (3) holds for interior as well as boundary points $\boldsymbol{x}$. If $p$ satisfies $p \in C^{0, \alpha}(\Gamma)$ at $\boldsymbol{x} \in \Gamma$, the regularizing effect of the factor $[p(\boldsymbol{y})-p(\boldsymbol{x})]$ is effective and all integrals in (3) are weakly singular. From (3), the direct problem (1) is then equivalent to the following 'primary' BIE:

$$
\begin{aligned}
& p(\boldsymbol{x})+\int_{\Gamma} p(\boldsymbol{y})\left[G_{, n}(\boldsymbol{x}, \boldsymbol{y})-G_{, n}^{0}(\boldsymbol{x}, \boldsymbol{y})\right] \mathrm{d} S_{y}+\int_{\Gamma}[p(\boldsymbol{y})-p(\boldsymbol{x})] G_{, n}^{0}(\boldsymbol{x}, \boldsymbol{y}) \mathrm{d} S_{y} \\
&=-\int_{\Gamma} p_{, n}^{I}(\boldsymbol{y}) G(\boldsymbol{x}, \boldsymbol{y}) \mathrm{d} S_{y}
\end{aligned}
$$

\section{Material differentiation of integral functionals}

To investigate the variations of $\mathcal{J}$ or other shape-dependent integral functionals, one has to consider the effect of small perturbations of $\Gamma$. The latter can be described by means of a normal transformation velocity $\theta$ on $\Gamma$, and using a small time-like parameter $\tau \geq 0$ :

$$
\boldsymbol{y} \in \Gamma \rightarrow \boldsymbol{y}+\theta(\boldsymbol{y}) \boldsymbol{n}(\boldsymbol{y}) \tau \quad \text { i.e. } \Gamma(\tau)=\Gamma+\theta \boldsymbol{n} \tau \quad(\tau \geq 0,|\theta \tau| \ll \operatorname{Diam}(\Gamma))
$$

Integrals over $\Omega^{-}$or $\Gamma$ are then treated as functions of the scalar parameter $\tau$ and differentiated accordingly at $\tau=0$. Indeed the material differentiation approach consists merely of the application of general results of continuum kinematics to situations where $\tau$ is not the physical time: eq. (5) describes a geometrical, non-physical, domain perturbation.

Several kinds of derivatives with respect to $\tau$ are available for fields $u(\boldsymbol{y}, \tau)$ ('eulerian' description of $u$ ). Owing to the BIE context, it looks appropriate to use 'material' derivatives on $\Gamma$, i.e. to 'follow' the value of $u(\boldsymbol{y}, \tau)$ while the field point $\boldsymbol{y} \in \Gamma$ moves according to (5), in order to keep things defined on the boundary alone. When, as in eq. (5), the geometrical transformation is described by means of a normal velocity $\theta \boldsymbol{n}$, the material derivative of $u$ reduces to the 'transformation derivative' $\stackrel{\star}{u}$ (Petryk \& Mroz [22]):

$$
\stackrel{\star}{u}(\boldsymbol{y}, 0)=\lim _{\tau \rightarrow 0} \frac{1}{\tau}[u(\boldsymbol{y}+\theta \boldsymbol{n} \tau, \tau)-u(\boldsymbol{y}, 0)]=u_{, \tau}(\boldsymbol{y}, 0)+u_{, n}(\boldsymbol{y}, 0) \theta(\boldsymbol{y})
$$

where $u_{, \tau}$ denotes the partial derivative of $u$ with respect to $\tau$, i.e. with $\boldsymbol{y}$ kept fixed. Obviously one has $\mathcal{J}=d \mathcal{J} / d \tau$ for functionals like (2). Various formulas are found in the literature [22] for the derivative of 
integrals with respect to volumes $\Omega(\tau)$ or surfaces $\Gamma(\tau)$ varying according to (5), among which:

$$
\begin{aligned}
\frac{d}{d \tau} \int_{\Omega(\tau)} a(\boldsymbol{y}, \tau) \mathrm{d} V_{y} & =\int_{\Omega} a_{, \tau}(\boldsymbol{y}, \tau) \mathrm{d} V_{y}+\int_{\Gamma} a(\boldsymbol{y}, \tau) \theta(\boldsymbol{y}) \mathrm{d} S_{y} \\
\frac{d}{d \tau} \int_{\Gamma(\tau)} a(\boldsymbol{y}, \tau) \mathrm{d} S_{y} & =\int_{\Gamma}\left\{a_{, \tau}(\boldsymbol{y}, \tau)+\left(a_{, n}(\boldsymbol{y}, \tau)-2 K(\boldsymbol{y}) a(\boldsymbol{y}, \tau)\right) \theta(\boldsymbol{y})\right\} \mathrm{d} S_{y} \\
& =\int_{\Gamma}\left\{{ }^{\star}(\boldsymbol{y}, \tau)-2 K(\boldsymbol{y}) a(\boldsymbol{y}, \tau) \theta(\boldsymbol{y})\right\} \mathrm{d} S_{y}
\end{aligned}
$$

where $K=\frac{1}{2} \operatorname{div}_{S} \boldsymbol{n}$ denotes the mean curvature at $\boldsymbol{y} \in \Gamma$. Equations (8), (9) hold only for a closed smooth surface, while in equation (7) $\theta$ refers to the unit normal $\boldsymbol{n}$ exterior to $\Omega$. Generalization of above formulas to piecewise smooth surfaces is available [22] but not needed here.

\section{The adjoint problem approach.}

Introduction of an augmented functional. The adjoint problem approach, known e.g. in the field of structural shape optimization (see [15], and also [11], [19] for applications in BIE context), basically consists in considering the minimization of $\mathcal{J}(\Gamma)$ as the minimization of $J(p)$ subject to the constraint $p=p_{\Gamma}$. The latter admits the following weak formulation:

$$
\mathcal{A}(p, w ; \Gamma) \equiv \int_{\Omega_{e}}\left(\nabla p \cdot \nabla \bar{w}-k^{2} p \bar{w}\right) \mathrm{d} V_{y}+\int_{\Gamma} \bar{w} p_{, n}^{I} \mathrm{~d} S_{y}=0 \quad(\forall w \in \mathcal{V})
$$

using the space of test functions $\mathcal{V}=\left\{w \in H_{\text {loc }}^{1}(\Omega)\right\}$. An augmented functional $\mathcal{L}$ is thus introduced:

$$
\mathcal{L}(p, w ; \Gamma)=J(p)+\mathcal{A}(p, w ; \Gamma)
$$

where the test function $w \in \mathcal{V}$ acts as a Lagrange multiplier. Application of formulas (7), (8) to (11) gives:

$$
\begin{aligned}
\stackrel{\mathcal{L}}{\mathcal{L}}= & \int_{C} p_{, \tau} \bar{\jmath}, p(p-\hat{p}) d C+\int_{\Omega_{e}}\left(\nabla p_{, \tau} \cdot \boldsymbol{\nabla} \bar{w}-k^{2} p_{, \tau} \bar{w}\right) \mathrm{d} V+\int_{\Gamma} \theta\left(\boldsymbol{\nabla} p \cdot \nabla \bar{w}-k^{2} p \bar{w}\right) \mathrm{d} S \\
& +\int_{\Gamma}\left\{\bar{w}\left[\left(p_{, n}^{I}\right)^{\star}-2 K p_{, n}^{I} \theta\right]+p_{, n}^{I} \bar{w}_{, n} \theta\right\} \mathrm{d} S+\mathcal{A}\left(p, w_{, \tau} ; \Gamma\right)
\end{aligned}
$$

Some simplifications can be made on the right-hand side of (12). First, since $w_{, \tau} \in \mathcal{V},(10)$ implies:

$$
\mathcal{A}\left(p, w_{\tau} ; \Gamma\right)=0
$$

Next, splitting the gradients into tangential and normal parts (see Appendix A) gives:

$$
\nabla p \cdot \nabla \bar{w}=\nabla_{S} p \cdot \nabla_{S} \bar{w}+p_{, n} \bar{w}_{, n}
$$

Also, the following identity is established in appendix B:

$$
\left(p_{, n}^{I}\right)^{\star}=\left(2 K p_{, n}^{I}-k^{2} p^{I}\right) \theta-\operatorname{div}_{S}\left(\theta \nabla_{S} p^{I}\right)
$$

Upon substitution of the last two equations in the last two integrals of (12), one gets:

$$
\begin{aligned}
& \int_{\Gamma} \theta\left(\boldsymbol{\nabla} p \cdot \boldsymbol{\nabla} \bar{w}-k^{2} p \bar{w}\right) \mathrm{d} S+\int_{\Gamma}\left\{\bar{w}\left[\left(p_{, n}^{I}\right)^{\star}-2 K p_{, n}^{I} \theta\right]+p_{, n}^{I} \bar{w}{ }_{, n} \theta\right\} \mathrm{d} S \\
& =\int_{\Gamma} \theta\left(\nabla_{S} p \cdot \nabla_{S} \bar{w}-k^{2} p \bar{w}\right) \mathrm{d} S+\int_{\Gamma}\left(p_{, n}+p_{, n}^{I}\right) \bar{w} \mathrm{~d} S-\int_{\Gamma}\left\{\operatorname{div}_{S}\left(\theta \nabla_{S} p^{I}\right)+\theta k^{2} p^{I}\right\} \bar{w} \mathrm{~d} S \\
& \left.=\int_{\Gamma} \theta\left\{\nabla_{S}\left(p+p^{I}\right) \cdot \nabla_{S} \bar{w}-k^{2}\left(p+p^{I}\right) \bar{w}\right)\right\} \mathrm{d} S
\end{aligned}
$$


where the boundary conditions $(1)_{2}$ has been used and identity (69) for integration by parts has been applied with $f \equiv \bar{w} \theta \nabla_{S} p^{I}$. Finally, using (13) and (15) and taking into account the constraint $p=p_{\Gamma}$, equation (12) becomes:

$$
\begin{aligned}
\stackrel{\star}{\mathcal{L}} & =\mathcal{L}_{, p} p_{, \tau}+\mathcal{L}_{, \Gamma} \theta \\
\mathcal{L}_{, p} p_{, \tau} & =\int_{C} p_{, \tau} \bar{\jmath}_{, p}\left(p_{\Gamma}-\hat{p}\right) d C+\int_{\Omega_{e}}\left(\nabla p_{, \tau} \cdot \nabla \bar{w}-k^{2} p_{, \tau} \bar{w}\right) \mathrm{d} V \\
\mathcal{L}_{, \Gamma} \theta & =\int_{\Gamma} \theta\left[\nabla_{S} \bar{w} \cdot \nabla_{S}\left(p_{\Gamma}+p^{I}\right)-k^{2} \bar{w}\left(p_{\Gamma}+p^{I}\right)\right] \mathrm{d} S
\end{aligned}
$$

Definition of the adjoint problem. Indeed one is interested here in the net effect of a domain perturbation, and expects actual variations of $\mathcal{L}$ only when $\theta \neq 0$. Hence the Lagrange multiplier $w$ can be chosen so that $(\theta=0) \Rightarrow(\stackrel{\star}{\mathcal{L}}=0)$. This defines an adjoint state $w_{\Gamma}$, solution to the variational problem:

$$
\mathcal{L}_{, p}\left(p_{\Gamma}, w ; \Gamma\right) p_{, \tau}=0, \quad \forall p_{, \tau} \in \mathcal{V}
$$

The strong formulation for the adjoint problem (19-17) above reads:

$$
\begin{cases}\left(\Delta+k^{2}\right) w=-\bar{\jmath}_{, p}\left(p_{\Gamma}-\hat{p}\right) \delta_{C} & \text { in } \Omega \\ w_{, n}=0 & \text { on } \Gamma \\ \text { (radiation condition) } & \end{cases}
$$

One notices that this is a homogeneous exterior Neumann problem for Helmholtz' equation with a nonzero internal source $F=-\bar{\jmath}_{, p}\left(p_{\Gamma}-\hat{p}\right) \delta_{C}$ proportional to the difference between measured and computed pressure on the measurement surface. For example, one has $F=-\left(\overline{p_{\Gamma}-\hat{p}}\right) \delta_{C}$ for the least-squares distance $2 j=$ $|p-\hat{p}|^{2}$.

Material derivative of $\mathcal{J}(\Gamma)$. Finally, from (16-19) the material derivative of $\mathcal{J}(\Gamma)$ is given in terms of $p_{\Gamma}, w_{\Gamma}, \theta$ by:

$$
\begin{aligned}
\stackrel{\star}{\mathcal{J}}(\Gamma) & =\stackrel{\star}{\mathcal{L}}\left(p_{\Gamma}, w_{\Gamma} ; \Gamma\right) \\
& =\int_{\Gamma} \theta\left[\nabla_{S} \bar{w}_{\Gamma} \cdot \nabla_{S}\left(p_{\Gamma}+p^{I}\right)-k^{2} \bar{w}_{\Gamma}\left(p_{\Gamma}+p^{I}\right)\right] \mathrm{d} S
\end{aligned}
$$

The above formula gives explicitly, in an elegant fashion, the gradient of $\mathcal{J}(\Gamma)$ with respect to $\Gamma$, and thus the value of the directional derivative of $\mathcal{J}(\Gamma)$ for a given obstacle perturbation velocity $\theta$. In more abstract terms, the domain derivative kernel $\mathcal{J}_{, \Gamma}$ is explicitly known, so that one has:

$$
\begin{aligned}
\mathcal{J}(\Gamma) & =\int_{\Gamma} \mathcal{J}_{, \Gamma}(\boldsymbol{y} ; \Gamma) \theta \mathrm{d} S \\
\mathcal{J}_{, \Gamma}(\boldsymbol{y} ; \Gamma) & =\nabla_{S} \bar{w}_{\Gamma}(\boldsymbol{y}) . \nabla_{S}\left(p_{\Gamma}+p^{I}\right)(\boldsymbol{y})-k^{2} \bar{w}_{\Gamma}(\boldsymbol{y})\left(p_{\Gamma}+p^{I}\right)(\boldsymbol{y})
\end{aligned}
$$

Gradient evaluation using BIE. The general acoustic BIE (3) gives, in view of (20), the following BIE formulation of the adjoint problem:

$$
\begin{aligned}
w(\boldsymbol{x})+\int_{\Gamma} w(\boldsymbol{y})\left[G_{, n}(\boldsymbol{x}, \boldsymbol{y})-G_{, n}^{0}(\boldsymbol{x}, \boldsymbol{y})\right] \mathrm{d} S_{y} & \\
& +\int_{\Gamma}[w(\boldsymbol{y})-w(\boldsymbol{x})] G_{, n}^{0}(\boldsymbol{x}, \boldsymbol{y}) \mathrm{d} S_{y}=\int_{C} \bar{\jmath}_{, p}\left(p_{\Gamma}-\hat{p}\right)(\boldsymbol{y}) G(\boldsymbol{x}, \boldsymbol{y}) \mathrm{d} C_{y}
\end{aligned}
$$


Note that, thanks to the presence of tangential derivatives, eq. (21) defines $\stackrel{\star}{\mathcal{J}}$ in terms of the boundary values of $p_{\Gamma}, p^{I}, w_{\Gamma}$. Hence it is directly computable as soon as the primary and adjoint BIEs, resp. (4) and (23), are solved for $p_{\Gamma}$ and $w_{\Gamma}$ respectively.

Generalization to $N$ incident waves. In the case where $\Omega^{-}$is illuminated by $N$ incident waves $p_{i}^{I}$ $(i=1, \ldots, N)$ in succession, generating $N$ scattered pressure fields $p_{\Gamma}^{i}$ in $\Omega$ and $N$ sets of measurements $\hat{p}^{i}$ on $C$, the cost function (2) becomes:

$$
\mathcal{J}(\Gamma)=\sum_{i=1}^{N} \int_{C} j\left(p_{\Gamma}^{i}-\hat{p}^{i}\right)^{2} \partial C
$$

The material derivative of $\mathcal{J}(\Gamma)$ is then given by:

$$
\stackrel{\star}{\mathcal{J}}=\sum_{i=1}^{N} \int_{\Gamma} \theta\left[\nabla_{S} \bar{w}_{\Gamma}^{i} \cdot \nabla_{S}\left(p_{\Gamma}^{i}+p_{i}^{I}\right)-k^{2} \bar{w}_{\Gamma}^{i}\left(p_{\Gamma}^{i}+p_{i}^{I}\right)\right] d S
$$

where the $w_{\Gamma}^{i}$ solve the $N$ adjoint problems:

$$
\begin{cases}\left(\Delta+k^{2}\right) w^{i}=-\bar{\jmath}_{, p}\left(p_{\Gamma}^{i}-\hat{p}^{i}\right) \delta_{C} & \text { in } \Omega \\ w_{, n}^{i}=0 & \text { on } \Gamma \\ \text { (radiation condition) } & \end{cases}
$$

Comments. The adjoint problem approach as presented here is clearly not specifically linked to BIE methods. The result (21) pertains to BIE analysis only to the extent that the primary and adjoint states can be formulated using BIE methods.

The adjoint state $w_{\Gamma}$ does not depend on $\theta$. Therefore the kernel $\mathcal{J}_{, \Gamma}(22)$, or equivalently any directional derivative $\mathcal{J}^{\star}(21)$, is computable once the primary and adjoint states are known, i.e. at the expense of only two BEM solutions (or, more generally, $(N+1)$ BEM solutions for the gradient of $N$ distinct functionals) over the same geometry. This is true whatever the number, finite or infinite, of design parameters. In contrast, the direct differentiation approach needs $(D+1)$ BEM solutions, $D$ being the number of design parameters, whatever the number of functionals present.

Moreover, the primary and adjoint problems are associated with the same partial differential, or integral, governing operator and boundary conditions of the same type (here, Neumann). Thus, the operator matrix, which must be built and factored in order to compute the primary state $p_{\Gamma}$ and evaluate $\mathcal{J}(\Gamma)$, is then reused to compute the adjoint state $w_{\Gamma}$ and the domain derivative kernel $\mathcal{J}_{, \Gamma}$.

\section{Numerical implementation and examples}

In this section, the numerical implementation for the solution of the 3D inverse problem using shape differention and the adjoint problem approach is presented. The regularized collocation BIE (3) is implemented in our BEM research code AstRID. The usual BE discretization of $\Gamma$ has been employed, using shape functions $N^{k}(\boldsymbol{\xi})$ associated with 8-noded curved elements and interpolation nodes $\boldsymbol{y}^{k}$. An isoparametric discretization 
for the boundary variables $p, w$ has been used. To represent the varying boundary $\Gamma$ the $\boldsymbol{y}^{k}$ nodes are known functions of $D$ design parameters $\boldsymbol{d}=\left(d_{1}, \ldots, d_{D}\right)$. Then, the BE parametrization of a point $\boldsymbol{y}$ on a typical $N$-noded element $E$ of parent element $E_{0}$ has the form:

$$
\boldsymbol{y}=\sum_{k=1}^{N} N^{k}(\boldsymbol{\xi}) \boldsymbol{y}^{k}(\boldsymbol{d}) \quad\left(\boldsymbol{\xi} \in E_{0}\right)
$$

This method amounts to monitoring the evolution of $\Gamma$ by moving the mesh nodes according to their known $\boldsymbol{d}$-dependency. The discretized normal velocity $\theta$ associated with the variation of a single parameter $d_{j}$ is then given by:

$$
\theta_{j}=\sum_{k=1}^{N} N^{k}(\boldsymbol{\xi})\left[\boldsymbol{y}_{, d_{j}}^{k}(\boldsymbol{d}) . \boldsymbol{n}\right] \quad\left(\boldsymbol{\xi} \in E_{0}\right)
$$

The partial derivative $\mathcal{J}_{d_{i}}$ is then computed using (21) with $\theta=\theta_{j}$ as defined in the above equation.

\subsection{Example with geometrical parameters as unknowns}

Parametrization of the unknown boundary. Here the unknown surface $\Gamma$ is searched as a 'superelliptic', or ' $n$-ellipsoidal', shape defined by 10 geometrical parameters: the centroid coordinates $x_{G}, y_{G}, z_{G}$, principal axes $a, b, c$, Euler angles $\phi, \theta, \psi$ of principal directions and the exponent $n(1 \leq n \leq+\infty)$ of the unit $n$-sphere $\mathcal{S}$ of equation:

$$
Y_{1}^{n}+Y_{2}^{n}+Y_{3}^{n}=1
$$

The current shape $\Gamma$ is then defined as an affine distortion of $\mathcal{S}$ :

$$
\boldsymbol{Y} \in \mathcal{S} \rightarrow \boldsymbol{y} \in \Gamma \quad\left\{\begin{array}{l}
y_{1}=x_{G}+r_{11} a Y_{1}+r_{12} b Y_{2}+r_{13} c Y_{3} \\
y_{2}=y_{G}+r_{21} a Y_{1}+r_{22} b Y_{2}+r_{23} c Y_{3} \\
y_{3}=y_{G}+r_{31} a Y_{1}+r_{32} b Y_{2}+r_{33} c Y_{3}
\end{array}\right.
$$

where $r_{i j}=r_{i j}(\phi, \theta, \psi)$ are the components of the rotation matrix which maps the coordinate axes onto the principal axes of the ellipsoid (several $(\phi, \theta, \psi)$ triplets may define the same rotation). In the numerical implementation, the analytical description (29) is used to define the dependency $\boldsymbol{y}^{k}=\boldsymbol{y}^{k}(\boldsymbol{d})$ of the nodes in eqs. (27), (28). For the special cases $n=1, n=2$ and $n=+\infty \mathcal{S}$ is respectively the regular octahedron of vertices $( \pm 1,0,0),(0, \pm 1,0),(0,0, \pm 1)$, the unit sphere and the unit cube of vertices $( \pm 1, \pm 1, \pm 1)$.

Minimization algorithm. Both conjugate gradient (CG) and BFGS Quasi-Newton algorithms have been initially applied to the minimization of $\mathcal{J}$, using software from Press et al. [23]. However, our first numerical evidence suggested that:

- CG and BFGS perform similarly in terms of convergence and accuracy, but BFGS is generally faster.

- The computational efficiency of the algorithm, and sometimes the level of convergence, is found to be strongly dependent on the line search algorithm imbedded in both CG and BFGS methods, and especially on the initialization of its 'bracketing' [14], [23] step. 
Thus, in a second stage, the line search algorithm provided by [23] has been replaced by an implementation of the method described in Fletcher [14]. The latter features a user-set parameter $\sigma \in] 0,1[$, which allows one to choose from high accuracy $(\sigma \sim 0)$ to low accuracy $(\sigma \sim 1)$ line searches. In our experience the best results, in terms of both accuracy and computational speed, were obtained using BFGS Quasi-Newton algorithm together with low-accuracy line search. It is interesting to note that a similar conclusion has been reached independently in [18], which deals with crack identification using potential theory. All results presented in this paper were produced using the latter algorithm, with $\sigma=.9$.

Description of the examples. Numerical results are presented below for six situations. The unknown boundary is made of 24 eight-noded elements and 74 nodes. For all examples, synthetic data were created for one $\left(p_{3}^{I}\right)$, two $\left(p_{2}^{I}, p_{3}^{I}\right)$ and three $\left(p_{1}^{I}, p_{2}^{I}, p_{3}^{I}\right)$ incident waves, where $p_{i}^{I}$ denotes the plane wave which propagates along $\boldsymbol{e}_{i}$ in the positive direction. In order to simulate the effect of measurement noise, the known values $\hat{p}$ have been multiplied by $(1+r)$, where $r$ are random numbers uniformly distributed in $[-\epsilon, \epsilon]$. the values $\epsilon=0$ (perfect data), $\epsilon=10^{-3}, 10^{-2}, 10^{-1}$ were used. The measurement surface $C$ is a sphere of radius 10 units, centered at the origin, meshed using 96 elements and 290 nodes. The known values $\hat{p}$ of $p$ at the 290 nodes were synthetic data, obtained from the solution of the direct problem (4) with the 'true' location of $\Gamma$ and then interpolated on $C$ using the usual isoparametric 8-noded shape functions for the numerical evaluation of integrals over $C$ in (2) or (23).

The 'true' and initial values of the design parameters are shown in table 1. For examples 1,2 the exponent $n$ is not included in the search (9 unknowns) and is given the value $n=2$ for both the 'true' and search surfaces. For examples 3 to 6 , the 'true' values of $\left(d_{1}, \ldots, d_{10}\right)$ are the same, but:

- The exponent $n=d_{10}$ is excluded from the search in examples 3,4 and included in examples 5,6 .

- The 'true' obstacle is an ellipsoid $(n=2)$ in examples 3,5 and a rectangular box $(n=+\infty)$ in examples 4,6 .

Thus, in example 4, the 'true' obstacle cannot be reached exactly by the minimization process.

\section{Table 1 should appear here.}

As the same ' $n$-ellipsoid' can result from many combinations of Euler angles and permutations of principal axes, the accuracy of the identification of $\Gamma$ cannot be measured by merely comparing the identified parameters $d_{k}$ with those defining the 'true' $\Gamma$ and used to compute the simulated data. Instead, the relative errors $e_{V}, e_{A}, e_{I}$ for the volume, boundary area and geometrical inertia tensor (with respect to a fixed coordinate system having no particular relation to $\Gamma$ ) of $\Omega^{-}$, given by:

$$
e_{V}=\frac{V\left(\Gamma_{n}\right)}{V(\Gamma)}-1 \quad e_{A}=\frac{A\left(\Gamma_{n}\right)}{A(\Gamma)}-1 \quad e_{I}=\left(\frac{\sum_{1 \leq i, j \leq 3}\left(I_{i j}\left(\Gamma_{n}\right)-I_{i j}(\Gamma)\right)^{2}}{\sum_{1 \leq i, j \leq 3} I_{i j}^{2}(\Gamma)}\right)^{1 / 2}
$$

with

$$
V(S)=\frac{1}{3} \int_{S} y_{i} n_{i} \mathrm{~d} S_{y} \quad A(S)=\int_{S} \mathrm{~d} S_{y} \quad I_{i j}(S)=\frac{1}{5} \int_{S} y_{i} y_{j} y_{k} n_{k} \mathrm{~d} S_{y}
$$


have been computed (the indicator $e_{I}$ being very sensitive to the orientation of $\Gamma$ in space), together with the relative decrease $\mathcal{J}_{\text {final }} / \mathcal{J}_{\text {initial }}$ of the cost function achieved by the minimization process.

Numerical results and discussion. Our numerical results for $\mathcal{J}_{\text {final }} / \mathcal{J}_{\text {initial }}, e_{V}, e_{A}, e_{I}$ obtained for examples 1 to 6 are displayed in tables $2,3,4,5,6,7$, together with the number of cost function and gradient evaluations spent during the minimization. Table 7 also shows the value of $d_{10}=n$ reached (the 'true' value being $n=\infty$ for this example).

Table 2 should appear here.

Table 3 should appear here.

Table 4 should appear here.

Table 5 should appear here.

Table 6 should appear here.

Table 7 should appear here.

Examples 1, 2, 3, 5, 6 exhibit very good convergence and accuracy, especially for non-perturbed data, see tables $2,3,4,6,7$. This is a clear indication of the good performance of the adjoint problem approach for the gradient evaluations. At least in the range $\epsilon=10^{-3}$ to $10^{-1}$, the error indicators $e_{V}, e_{A}, e_{I}$ are often found to vary linearly with $\epsilon$ in the results presented here, and $\mathcal{J}_{\text {final }} / \mathcal{J}_{\text {initial }}$ to vary quadratically. The numerical solution of the inverse problem hence behaves well with respect to measurement noise. This is likely to be a consequence of the strong assumption made on the unknown geometry, which is described using only 10 parameters.

The convergence and accuracy remains good for example 4 (table 5), where the 'true' cavity is a rectangular box and exact convergence is hence impossible. The 'final' ellipsoid found by the algorithm has very similar volume and inertia tensor than the box (see table 5) and slightly different area. Moreover, the results appear to be less sensitive to data noise than in the other examples, where the exact shape can be reached by the minimization.

Convergence is much slower when $d_{10} \equiv n$ plays an active role and is included in the search, see table 7 . When only one incident wave is considered, the recovery of $n$ in example 6 is very much affected by measurement noise. Moreover, the recovery of $n$ has been found to be sensitive to implementation details like how the BFGS updating formula is written or the initialization of the line-search. This suggests that the recovery of $n$ is a more ill-posed problem than the recovery of Euler angles, principal axes and center coordinates.

Upon comparison of the convergence process for examples 4 and 6 (i.e. search of a box with $n$ respectively excluded and included), it has been noticed that they are almost identical until the example 4 termination. In view of the respective function/gradient evaluation counts for examples 4 and 6 , one concludes that a large amount (about two-thirds) of computing effort in example 6 is spent to recover $n$ alone. 
All numerical computations presented here have been done in double precision complex arithmetic, on HP-Apollo 400-type workstations. The overall computer time spent for solving the inverse problem varies from about 15 to 75 minutes. The determination of the exponent $n$ is time-consuming. Every cost function and gradient evaluation, using the procedure described in section 4, takes about 20 seconds, with about 15 seconds spent on $\mathcal{J}$ alone; thus gradient evaluations using the adjoint problem approach are relatively inexpensive.

The efficiency of the line search is an important issue since here most computer time is spent on cost function and gradient evaluation. Here, using low-accuracy line searches, most BFGS iterations used only one evaluation.

\subsection{Example with nodal coordinates as unknowns}

The previous examples used a moderate number of design parameters, which implies obvious limitations on the allowed shapes. One may think about using directly the mesh nodes as design variables. Ideally one would have to introduce one scalar design variable per node (e.g. the value taken at each node by the normal velocity $\theta$ ). However this raises some technical difficulties for $3 \mathrm{D}$ geometries because $\Gamma$ is only $C^{0, \alpha}$ at the nodes. One can also assume restrict the study to star-shaped obstacles and measure the node locations along rays emanating from an origin point. A similar idea has been implemented by Kassab et al. [16]; Nishimura and Kobayashi also propose an interesting parametrization idea for crack inverse problems [21] (both references deal with 2D problems).

An example (referred to as example 7) is now shown where the three coordinates of the BE mesh nodes are used as design parameters, with no prior information on the unknown shape. In view of previous comments this choice of representation is not good but has nonetheless be tried in view of its simplicity of implementation. The true and initial data are those of example 1, see table 1. Note that this initial guess is much closer to the solution than in the examples 2 to 6 previously discussed. The converged values of $\mathcal{J}_{\text {final }} / \mathcal{J}_{\text {initial }}, e_{V}, e_{A}, e_{I}$, using the 222 nodal coordinates or the 9 parameters $\left(y_{1}^{G}, y_{2}^{G}, y_{3}^{G}, a, b, c, \phi, \theta, \psi\right)$ as unknowns, are given in table 8. It is seen that the values obtained for $\mathcal{J}_{\text {final }} / \mathcal{J}_{\text {initial }}, e_{V}, e_{I}$ with nodes as unknowns are not as good as in the 9 unknowns example; they nevertheless are reasonable and show that the location, size and orientation of the obstacle are correctly reconstructed. On the contrary, the final value of about $34 \%$ taken by $e_{A}$ indicates strong oscillations of the reconstructed surface, which is typical of ill-posed problems.

Table 8 should appear here.

\section{Adjoint problem approach: extension to penetrable obstacles}

Direct problem for a penetrable obstacle. The scatterer $\Omega^{-}$is now assumed to be an acoustical medium characterized by a wave number $k^{-} \neq k$ and a mass density $\rho^{-} \neq \rho$. The direct problem for the 
scattered pressure $p$ induced by the presence of the penetrable obstacle $\Omega^{-}$is:

$$
\begin{cases}\Delta p+k^{2} p=0 & \text { in } \Omega \\ \Delta p+k_{-}^{2} p=\left(k^{2}-k_{-}^{2}\right) p^{I} & \text { in } \Omega^{-} \\ p-p^{-}=0 & \text { on } \Gamma \\ p_{, n}^{-}=a p_{, n}+(a-1) p_{, n}^{I} & \text { on } \Gamma \\ \text { (radiation condition) } & \end{cases}
$$

where the known incident wave $p^{I}$ is such that $\left(\Delta+k^{2}\right) p^{I}=0$ in $\mathbb{R}^{3}$ and is assumed to be continuous together with its gradient accross $\Gamma$. The notation $f^{-}$refers to the limit on the boundary $\Gamma$ 'from inside', i.e. of variables $f$ defined in the interior domain $\Omega^{-}$:

$$
f^{-}(\boldsymbol{y})=\lim _{\epsilon \searrow 0} f(\boldsymbol{y}+\epsilon \boldsymbol{n}) \quad(\boldsymbol{y} \in \Gamma)
$$

while $f$ refers to the limit on $\Gamma$ 'from outside'. The solution to the system (30) is denoted $p_{\Gamma}$. The boundary condition $(30)_{4}$ expresses the velocity continuity accross $\Gamma ; a$ denotes the ratio $\rho^{-} / \rho$.

Coupled BIE formulation. The direct problem (30) can be formulated, using (3), in terms of two coupled regularized BIEs on the two independent boundary unknowns $\left.\left(p, p_{n}\right)\right|_{\Gamma}$ which remain after accounting for the boundary conditions $(30)_{3,4}$, as follows:

$$
\begin{aligned}
& p(\boldsymbol{x})+\int_{\Gamma} p(\boldsymbol{y})\left[G_{, n}(\boldsymbol{x}, \boldsymbol{y})-G_{, n}^{0}(\boldsymbol{x}, \boldsymbol{y})\right] \mathrm{d} S_{y} \\
& \quad+\int_{\Gamma}[p(\boldsymbol{y})-p(\boldsymbol{x})] G_{, n}^{0}(\boldsymbol{x}, \boldsymbol{y}) \mathrm{d} S_{y}-\int_{\Gamma} p_{, n}(\boldsymbol{y}) G(\boldsymbol{x}, \boldsymbol{y}) \mathrm{d} S_{y}=0 \\
& \int_{\Gamma} p(\boldsymbol{y})\left[G_{, n}^{-}(\boldsymbol{x}, \boldsymbol{y})-G_{, n}^{0}(\boldsymbol{x}, \boldsymbol{y})\right] \mathrm{d} S_{y}+\int_{\Gamma}[p(\boldsymbol{y})-p(\boldsymbol{x})] G_{, n}^{0}(\boldsymbol{x}, \boldsymbol{y}) \mathrm{d} S_{y} \\
& \quad-\int_{\Gamma}\left(a p_{, n}+(a-1) p_{, n}^{I}\right)(\boldsymbol{y}) G^{-}(\boldsymbol{x}, \boldsymbol{y}) \mathrm{d} S_{y}+\left(k^{2}-k_{-}^{2}\right) \int_{\Omega^{-}} G^{-}(\boldsymbol{x}, \boldsymbol{y}) p^{I}(\boldsymbol{y}) \mathrm{d} V_{y}=0
\end{aligned}
$$

where $G^{-}(\boldsymbol{x}, \boldsymbol{y})$ is the dynamic fundamental solution associated with the obstacle medium (wavenumber $\left.k_{-}\right)$.

Adjoint problem. The method of section 4 is generalized to the penetrable obstacle case, using similar notations. Following again the constrained minimization viewpoint, one introduces the augmented functional $\mathcal{L}$ as follows:

$$
\begin{aligned}
\mathcal{L}(p, w ; \Gamma)= & J(p)+\mathcal{A}(p, w ; \Gamma) \\
\mathcal{A}(p, w ; \Gamma) \equiv & \int_{\Omega}\left(\boldsymbol{\nabla} p \cdot \nabla \bar{w}-k^{2} p \bar{w}\right) \mathrm{d} V+\frac{1}{a} \int_{\Omega^{-}}\left(\boldsymbol{\nabla} p \cdot \nabla \bar{w}-k_{-}^{2} p \bar{w}\right) \mathrm{d} V \\
& +\frac{1}{a}\left(k^{2}-k_{-}^{2}\right) \int_{\Omega^{-}} p^{I} \bar{w} \mathrm{~d} V+\left(1-\frac{1}{a}\right) \int_{\Gamma} p_{, n}^{I} \bar{w} \mathrm{~d} S
\end{aligned}
$$


The test function $w \in \mathcal{V}$ is the Lagrange multiplier, with $\mathcal{V}=\left\{w \in H_{l o c}^{1}(\Omega), w=w^{-}\right.$on $\left.\Gamma\right\}$. Following calculations similar to those of section 4 , one obtains:

$$
\begin{aligned}
\stackrel{\star}{\mathcal{L}}= & \mathcal{L}_{, p} p_{, \tau}+\mathcal{L}_{, \Gamma} \theta \\
\mathcal{L}_{, p} p_{, \tau}= & \int_{C} p_{, \tau}\left(p_{\Gamma}-\hat{p}\right) d C+\int_{\Omega}\left(\boldsymbol{\nabla} p_{, \tau} \cdot \boldsymbol{\nabla} \bar{w}-k^{2} p_{, \tau} \bar{w}\right) \mathrm{d} V+\frac{1}{a} \int_{\Omega^{-}}\left(\boldsymbol{\nabla} p_{, \tau} \cdot \boldsymbol{\nabla} \bar{w}-k_{-}^{2} p_{, \tau} \bar{w}\right) \mathrm{d} V \\
\mathcal{L}_{, \Gamma} \theta= & \left(1-\frac{1}{a}\right) \int_{\Gamma} \theta \boldsymbol{\nabla} p \cdot \boldsymbol{\nabla} \bar{w} \mathrm{~d} S-\left(k^{2}-\frac{1}{a} k_{-}^{2}\right) \int_{\Gamma} \theta p \bar{w} \mathrm{~d} S \\
& -\frac{1}{a}\left(k^{2}-k_{-}^{2}\right) \int_{\Gamma} p^{I} \bar{w} \theta \mathrm{d} S+\left(1-\frac{1}{a}\right) \int_{\Gamma}\left\{\left(p_{, n}^{I}\right)^{\star} \bar{w}+\left(p_{, n}^{I} \bar{w}_{, n}-2 K p^{I} \bar{w}\right) \theta(\boldsymbol{y})\right\} \mathrm{d} S \\
= & \int_{\Gamma} \theta\left\{\left(1-\frac{1}{a}\right) \nabla_{S}\left(p+p^{I}\right) \cdot \nabla_{S} \bar{w}-\left(k^{2}-\frac{1}{a} k_{-}^{2}\right)\left(p+p^{I}\right) \bar{w}\right\} \mathrm{d} S
\end{aligned}
$$

where identity (14) and the boundary condition $(30)_{4}$ has been used. Moreover the adjoint problem, still defined by (19), has the following strong formulation:

$$
\begin{cases}\Delta w+k^{2} w=-\bar{\jmath}_{, p}\left(p_{\Gamma}-\hat{p}\right) \delta_{C} & \text { in } \Omega \\ \Delta w+k_{-}^{2} w=0 & \text { in } \Omega^{-} \\ w=w^{-} & \text {on } \Gamma \\ w_{, n}^{-}=a w_{, n} & \text { on } \Gamma \\ \text { (radiation condition) } & \end{cases}
$$

The two independent unknowns $\left.(w, w, n)\right|_{\Gamma}$ solve the following coupled BIE formulation:

$$
\begin{aligned}
& w(\boldsymbol{x})+\int_{\Gamma} w(\boldsymbol{y})\left[G_{, n}(\boldsymbol{x}, \boldsymbol{y})-G_{, n}^{0}(\boldsymbol{x}, \boldsymbol{y})\right] \mathrm{d} S_{y} \\
&+\int_{\Gamma}[w(\boldsymbol{y})-w(\boldsymbol{x})] G_{, n}^{0}(\boldsymbol{x}, \boldsymbol{y}) \mathrm{d} S_{y}-\int_{\Gamma} w_{, n}(\boldsymbol{y}) G(\boldsymbol{x}, \boldsymbol{y}) \mathrm{d} S_{y}=0 \\
& \int_{\Gamma} w(\boldsymbol{y})\left[G_{, n}^{-}(\boldsymbol{x}, \boldsymbol{y})-G_{, n}^{0}(\boldsymbol{x}, \boldsymbol{y})\right] \mathrm{d} S_{y} \\
& \quad+\int_{\Gamma}[w(\boldsymbol{y})-w(\boldsymbol{x})] G_{, n}^{0}(\boldsymbol{x}, \boldsymbol{y}) \mathrm{d} S_{y}-\int_{\Gamma} a w_{, n}(\boldsymbol{y}) G^{-}(\boldsymbol{x}, \boldsymbol{y}) \mathrm{d} S_{y}=0
\end{aligned}
$$

Domain derivative. The domain derivative kernel $\mathcal{J}_{, \Gamma}$ is given by:

$$
\begin{aligned}
\stackrel{\star}{\mathcal{J}} & =\int_{\Gamma} \mathcal{J}_{\Gamma} \theta \mathrm{d} S \\
\mathcal{J}_{, \Gamma} & =\left(1-\frac{1}{a}\right) \nabla_{S}\left(p+p^{I}\right) \cdot \nabla_{S} \bar{w}-\left(k^{2}-\frac{1}{a} k_{-}^{2}\right)\left(p+p^{I}\right) \bar{w}
\end{aligned}
$$

Note that taking the limiting case $\rho^{-} \rightarrow \infty$, i.e. $a \rightarrow \infty$, in the results (34), (35), (38) yields the corresponding ones for the hard obstacle given in Section 4 .

\section{Adjoint problem approach: extension to elastodynamics}

Elastodynamic direct problem. Here $\Omega^{-}$denotes a cavity embedded in an infinite elastic body (Poisson ratio $\nu$, shear modulus $\mu$ ), with a traction-free boundary $\Gamma=\partial \Omega$. The incident wave is an elastodynamic displacement $\boldsymbol{u}^{I}$ such that $\operatorname{div} \boldsymbol{\sigma}\left(\boldsymbol{u}^{I}\right)+\rho \omega^{2} \boldsymbol{u}^{I}=\mathbf{0}$. The scattered and total displacement fields $\boldsymbol{u}, \boldsymbol{u}^{T}$ are 
related by $\boldsymbol{u}^{T}=\boldsymbol{u}^{I}+\boldsymbol{u}$. The governing equations for the primary state $\boldsymbol{u}$ are:

$$
\left\{\begin{array}{cc}
\operatorname{div} \boldsymbol{\sigma}(\boldsymbol{u})+\rho \omega^{2} \boldsymbol{u}=\mathbf{0} & \text { in } \Omega \\
\boldsymbol{T}^{n}(\boldsymbol{u})+\boldsymbol{T}^{n}\left(\boldsymbol{u}^{\boldsymbol{I}}\right)=\mathbf{0} & \text { on } \Gamma \\
\text { (radiation conditions) } &
\end{array}\right.
$$

where

$$
\sigma_{i j}(\boldsymbol{u})=\mu\left(\frac{2 \nu}{1-2 \nu} \delta_{i j} u_{k, k}+u_{i, j}+u_{j, i}\right)
$$

is the elastic stress tensor associated with $\boldsymbol{u}$ and $\boldsymbol{T}^{n}(\boldsymbol{u})=\boldsymbol{\sigma}(\boldsymbol{u}) \cdot \boldsymbol{n}$ denotes the traction vector on $\Gamma$. The solution to the above system is denoted $\boldsymbol{u}_{\Gamma}$.

Inverse problem. Suppose that $\Gamma$ is unknown but $\boldsymbol{u}$ has known values $\hat{\boldsymbol{u}}$ on a measurement surface. The reconstruction of $\Gamma$ from the known values of $\hat{\boldsymbol{u}}$ can be attempted by minimizing a best-fit functional $\mathcal{J}(\Gamma)$ similar to (2):

$$
\mathcal{J}(\Gamma)=J\left(\boldsymbol{u}_{\Gamma}\right) \quad \text { with } \quad J(\boldsymbol{u})=\int_{C} j(\boldsymbol{u}) \mathrm{d} S
$$

Adjoint problem. Following again the approach developed in section 4, the following augmented functional is introduced:

$$
\begin{aligned}
\mathcal{L}(\boldsymbol{u}, \boldsymbol{w} ; \Gamma) & =J(\boldsymbol{u})+\mathcal{A}(\boldsymbol{u}, \boldsymbol{w} ; \Gamma) \\
\mathcal{A}(\boldsymbol{u}, \boldsymbol{w} ; \Gamma) & =\int_{\Omega}\left[\boldsymbol{\sigma}(\boldsymbol{u}): \boldsymbol{\nabla} \overline{\boldsymbol{w}}-\rho \omega^{2} \boldsymbol{u} \cdot \overline{\boldsymbol{w}}\right] \mathrm{d} V+\int_{\Gamma} \boldsymbol{T}^{n}\left(\boldsymbol{u}^{\boldsymbol{I}}\right) \cdot \boldsymbol{w} \mathrm{d} S
\end{aligned}
$$

The vector test function $\boldsymbol{w} \in \mathcal{V}$ is the Lagrange multiplier, with $\mathcal{V}=\left\{\boldsymbol{w} \in\left\{H_{l o c}^{1}(\Omega)\right\}^{3}\right\}$. Then, taking the material derivative of (41), one has:

$$
\begin{aligned}
\stackrel{\star}{\mathcal{L}}(\boldsymbol{u}, \boldsymbol{w} ; \Gamma)= & \mathcal{L}_{, u} \cdot \boldsymbol{u}_{, \tau}+\mathcal{L}_{, \Gamma} \cdot \theta \\
\mathcal{L}_{, u} \cdot \boldsymbol{u}_{, \tau}= & \int_{C} j_{, u} \cdot \boldsymbol{u}_{, \tau} \mathrm{d} S+\int_{\Omega}\left[\boldsymbol{\sigma}(\boldsymbol{u}, \tau): \nabla \overline{\boldsymbol{w}}-\rho \omega^{2} \boldsymbol{u}_{, \tau} \cdot \overline{\boldsymbol{w}}\right] \mathrm{d} S \\
\mathcal{L}_{, \Gamma} \cdot \theta= & \int_{\Gamma}\left[\boldsymbol{\sigma}(\boldsymbol{u}): \boldsymbol{\nabla} \overline{\boldsymbol{w}}-\rho \omega^{2} \boldsymbol{u} \cdot \overline{\boldsymbol{w}}\right] \theta \mathrm{d} S \\
& +\int_{\Gamma}\left\{\left(\boldsymbol{T}^{n}\left(\boldsymbol{u}^{\boldsymbol{I}}\right)\right)^{\star} \cdot \overline{\boldsymbol{w}}+\theta\left[\boldsymbol{T}^{n}\left(\boldsymbol{u}^{\boldsymbol{I}}\right) \cdot \overline{\boldsymbol{w}}_{, n}-2 K \boldsymbol{T}^{n}\left(\boldsymbol{u}^{\boldsymbol{I}}\right) \cdot \overline{\boldsymbol{w}}\right]\right\} \mathrm{d} S
\end{aligned}
$$

Equation (42) holds whatever the test function $\boldsymbol{w} \in \mathcal{V}$. Among those, the particular $\boldsymbol{w}$ which solves:

$$
\forall \boldsymbol{u}_{\tau} \in \mathcal{V} \quad \mathcal{L}_{, u}\left(\boldsymbol{u}_{\Gamma}, \boldsymbol{w} ; \Gamma\right) \cdot \boldsymbol{u}_{, \tau}=0
$$

is the adjoint state. In view of eq. (43) and since $\boldsymbol{u} \in \mathcal{V} \Rightarrow \boldsymbol{u}_{, \tau} \in \mathcal{V}$, the above variational equation defines a well-posed elastodynamic problem, whose solution, the adjoint state, is denoted $\boldsymbol{w}_{\Gamma}$. The strong formulation of the adjoint problem reads:

$$
\begin{cases}\operatorname{div} \boldsymbol{\sigma}(\boldsymbol{w})+\rho \omega^{2} \boldsymbol{w}=-j,{ }_{,} \delta_{C} & \text { in } \Omega \\ \boldsymbol{T}^{n}(\boldsymbol{w})=\mathbf{0} & \text { on } \Gamma \\ \text { (Radiation conditions) } & \end{cases}
$$


As a consequence, the derivative of $\mathcal{J}$ is given by:

$$
\begin{aligned}
\stackrel{\mathcal{J}}{\star}= & \mathcal{L}_{, \Gamma}\left(\boldsymbol{u}_{\Gamma}, \boldsymbol{w}_{\Gamma} ; \Gamma\right) \cdot \theta \\
= & \int_{\Gamma}\left[\boldsymbol{\sigma}\left(\boldsymbol{u}_{\Gamma}\right): \nabla \overline{\boldsymbol{w}}_{\Gamma}-\rho \omega^{2} \boldsymbol{u}_{\Gamma} \cdot \overline{\boldsymbol{w}}_{\Gamma}\right] \theta \mathrm{d} S \\
& \quad+\int_{\Gamma}\left\{\left(\boldsymbol{T}^{n}\left(\boldsymbol{u}^{\boldsymbol{I}}\right)\right)^{\star} \cdot \overline{\boldsymbol{w}}_{\Gamma}+\theta\left[\boldsymbol{T}^{n}\left(\boldsymbol{u}^{\boldsymbol{I}}\right) \cdot \overline{\boldsymbol{w}}_{\Gamma, n}-2 K \boldsymbol{T}^{n}\left(\boldsymbol{u}^{\boldsymbol{I}}\right) \cdot \overline{\boldsymbol{w}}_{\Gamma}\right]\right\} \mathrm{d} S
\end{aligned}
$$

The above formula involves the complete gradients of $\boldsymbol{u}_{\Gamma}, \boldsymbol{w}_{\Gamma}$, which is impractical. We then derive an alternative expression in terms of only the tangential gradients of $\boldsymbol{u}_{\Gamma}, \boldsymbol{w}_{\Gamma}$. This step makes use of the decomposition:

$$
\boldsymbol{\nabla} \boldsymbol{v}=\nabla_{S} \boldsymbol{v}+\boldsymbol{v}_{, n} \otimes \boldsymbol{n}
$$

Moreover, one has:

$$
\begin{aligned}
\left(\boldsymbol{T}^{n}\left(\boldsymbol{u}^{\boldsymbol{I}}\right)\right)^{\star} \cdot \boldsymbol{n} & =\left(\boldsymbol{\sigma}\left(\boldsymbol{u}^{I}\right)\right)^{\star}+\boldsymbol{\sigma}\left(\boldsymbol{u}^{I}\right) \cdot \stackrel{\star}{\boldsymbol{n}} \\
& =\left(\left\{\boldsymbol{\sigma}\left(\boldsymbol{u}^{I}\right)\right\}_{, \tau}+\theta\left\{\boldsymbol{\sigma}\left(\boldsymbol{u}^{I}\right)\right\}_{, n}\right) \cdot \boldsymbol{n}-\boldsymbol{\sigma}\left(\boldsymbol{u}^{I}\right) \cdot \nabla_{S} \theta
\end{aligned}
$$

Also, from the decomposition (48), one has for any stress tensor $\boldsymbol{\sigma}$ satisfying the dynamic equilibrium equation $(39)_{1}$ for some displacement $\boldsymbol{u}$ :

$$
\operatorname{div} \boldsymbol{\sigma}=\operatorname{div}_{S} \boldsymbol{\sigma}+\boldsymbol{\sigma}_{, n} \cdot \boldsymbol{n}=\rho \omega^{2} \boldsymbol{u}
$$

hence

$$
\boldsymbol{\sigma}_{, n} \cdot \boldsymbol{n}=-\operatorname{div}_{S} \boldsymbol{\sigma}-\rho \omega^{2} \boldsymbol{u}
$$

Substitution of (50) into (49) then yields:

$$
\begin{aligned}
\left(\boldsymbol{T}^{n}\left(\boldsymbol{u}^{\boldsymbol{I}}\right)\right)^{\star} & =\left\{\boldsymbol{\sigma}\left(\boldsymbol{u}^{I}\right)\right\}_{, \tau} \cdot \boldsymbol{n}-\theta \operatorname{div}_{S} \boldsymbol{\sigma}\left(\boldsymbol{u}^{I}\right)-\boldsymbol{\sigma}\left(\boldsymbol{u}^{I}\right) \cdot \nabla_{S} \theta-\rho \omega^{2} \boldsymbol{u}^{I} \\
& =-\operatorname{div}_{S}(\theta \boldsymbol{\sigma})-\rho \omega^{2} \boldsymbol{u}^{I}
\end{aligned}
$$

where the fact that $\boldsymbol{u}_{, \tau}^{I}=\mathbf{0}$ for the incident wave has been used. Next, the decomposition (48) and the above formula are substituted in (47), which becomes:

$$
\begin{aligned}
\mathcal{L}_{, \Gamma}\left(\boldsymbol{u}_{\Gamma}, \boldsymbol{w}_{\Gamma} ; \Gamma\right) \cdot \theta \\
=\int_{\Gamma}\left[\boldsymbol{\sigma}\left(\boldsymbol{u}_{\Gamma}\right): \nabla_{S} \overline{\boldsymbol{w}}_{\Gamma}+\boldsymbol{T}^{n}\left(\boldsymbol{u}_{\Gamma}\right) \cdot \overline{\boldsymbol{w}}_{\Gamma, n}-\rho \omega^{2} \boldsymbol{u}_{\Gamma} \cdot \overline{\boldsymbol{w}}_{\Gamma}\right] \theta \mathrm{d} S \\
\quad+\int_{\Gamma} \theta\left[\boldsymbol{T}^{n}\left(\boldsymbol{u}^{\boldsymbol{I}}\right) \cdot \overline{\boldsymbol{w}}_{\Gamma, n}-\rho \omega^{2} \boldsymbol{u}^{I} \cdot \overline{\boldsymbol{w}}_{\Gamma}\right] \mathrm{d} S-\int_{\Gamma}\left[\operatorname{div}_{S}\left(\theta \boldsymbol{\sigma}\left(\boldsymbol{u}^{I}\right)\right)+2 K \theta \boldsymbol{T}^{n}\left(\boldsymbol{u}^{\boldsymbol{I}}\right)\right] \cdot \overline{\boldsymbol{w}}_{\Gamma} \mathrm{d} S \\
=\int_{\Gamma}\left[\boldsymbol{\sigma}\left(\boldsymbol{u}_{\Gamma}+\boldsymbol{u}^{I}\right): \nabla_{S} \overline{\boldsymbol{w}}_{\Gamma}+\boldsymbol{T}^{n}\left(\boldsymbol{u}_{\Gamma}+\boldsymbol{u}^{\boldsymbol{I}}\right) \cdot \overline{\boldsymbol{w}}_{\Gamma, n}-\rho \omega^{2}\left(\boldsymbol{u}_{\Gamma}+\boldsymbol{u}^{I}\right) \cdot \overline{\boldsymbol{w}}_{\Gamma}\right] \theta \mathrm{d} S \\
=\int_{\Gamma}\left[\boldsymbol{\sigma}\left(\boldsymbol{u}_{\Gamma}+\boldsymbol{u}^{I}\right): \nabla_{S} \overline{\boldsymbol{w}}_{\Gamma}-\rho \omega^{2}\left(\boldsymbol{u}_{\Gamma}+\boldsymbol{u}^{I}\right) \cdot \overline{\boldsymbol{w}}_{\Gamma}\right] \theta \mathrm{d} S
\end{aligned}
$$

Use has been made of the integration by parts formula (69) and of the boundary condition (39) $)_{2}$ associated with the primary problem. The formula above is compact and very similar in structure to (38). Note that, contrary to the similar results $(22),(38)$ in acoustics, the above formula is still not directly expressed in terms of boundary variables or their tangential derivatives due to the presence of the complete tensor $\boldsymbol{\sigma}\left(\boldsymbol{u}+\boldsymbol{u}^{I}\right)$. 
However, expressing $\boldsymbol{\sigma}(\boldsymbol{u})$ in terms of $\boldsymbol{u}_{, n}$ and $\boldsymbol{T}^{n}(\boldsymbol{u})$ is a classical problem, which is briefly recalled in [6], and after some manipulation one can show that:

$$
\begin{aligned}
& \boldsymbol{\sigma}(\boldsymbol{u}): \nabla_{S} \overline{\boldsymbol{w}} \\
& =\boldsymbol{n} \cdot \nabla_{S} \overline{\boldsymbol{w}} \cdot \boldsymbol{T}^{n}(\boldsymbol{u})+\frac{\nu}{1-\nu}\left(\operatorname{div}_{S} \overline{\boldsymbol{w}}\right) \boldsymbol{T}^{n}(\boldsymbol{u}) \cdot \boldsymbol{n} \\
& \quad+\mu\left\{\frac{2 \nu}{1-\nu}\left(\operatorname{div}_{S} \boldsymbol{u}\right)\left(\operatorname{div}_{S} \overline{\boldsymbol{w}}\right)+\left(\nabla_{S} \boldsymbol{u}+{ }^{T} \boldsymbol{\nabla}_{S} \boldsymbol{u}\right): \nabla_{S} \overline{\boldsymbol{w}}-\left(\boldsymbol{n} \cdot \nabla_{S} \boldsymbol{u}\right) \cdot\left(\boldsymbol{n} \cdot \boldsymbol{\nabla}_{S} \overline{\boldsymbol{w}}\right)\right\}
\end{aligned}
$$

Then, accounting for the boundary condition $(39)_{2}$, one has:

$$
\begin{aligned}
\boldsymbol{\sigma}\left(\boldsymbol{u}+\boldsymbol{u}^{I}\right): \nabla_{S} \overline{\boldsymbol{w}} & =\mu\left\{\frac{2 \nu}{1-\nu}\left(\operatorname{div}_{S}\left(\boldsymbol{u}+\boldsymbol{u}^{I}\right)\right)\left(\operatorname{div}_{S} \overline{\boldsymbol{w}}\right)+\left(\nabla_{S}\left(\boldsymbol{u}+\boldsymbol{u}^{I}\right)+{ }^{T} \nabla_{S}\left(\boldsymbol{u}+\boldsymbol{u}^{I}\right)\right): \nabla_{S} \overline{\boldsymbol{w}}\right. \\
& \left.-\left(\boldsymbol{n} \cdot \nabla_{S}\left(\boldsymbol{u}+\boldsymbol{u}^{I}\right)\right) \cdot\left(\boldsymbol{n} \cdot \nabla_{S} \overline{\boldsymbol{w}}\right)\right\}
\end{aligned}
$$

in which only tangential derivatives are involved. The derivative (52) can thus be easily computed from the values of $\boldsymbol{u}_{\Gamma}, \boldsymbol{u}^{I}, \boldsymbol{w}_{\Gamma}$ on the boundary $\Gamma$.

\section{The direct differentiation approach}

The direct differentiation approach is applicable to both the acoustic and elastodynamic situations; we now briefly discuss it for the acoustic case. It basically consists of taking directly the material derivative of $\mathcal{J}(\Gamma)$ using eqn. (9). Since the measurement area $C$ is kept fixed, i.e. $\theta=0$ on $C$, this yields:

$$
\stackrel{\star}{\mathcal{J}}=\int_{C} \bar{\jmath}_{, p}\left(p_{\Gamma}-\hat{p}\right) p_{\Gamma}^{\star} \mathrm{d} C_{y}
$$

The derivative $p_{\Gamma}^{\star}$ in turn solves a governing derivative BIE, which is now established. First, note that the incident wave $p^{I}$ satisfies $\left(\Delta+k^{2}\right) p^{I}=0$ inside $\Omega^{-}$, and hence the interior BIE:

$$
\begin{gathered}
\int_{\Gamma} p^{I}(\boldsymbol{y})\left[G_{, n}(\boldsymbol{x}, \boldsymbol{y})-G_{, n}^{0}(\boldsymbol{x}, \boldsymbol{y})\right] \mathrm{d} S_{y}+\int_{\Gamma}\left[p^{I}(\boldsymbol{y})-p^{I}(\boldsymbol{x})\right] G_{, n}^{0}(\boldsymbol{x}, \boldsymbol{y}) \mathrm{d} S_{y} \\
=\int_{\Gamma} p_{, n}^{I}(\boldsymbol{y}) G(\boldsymbol{x}, \boldsymbol{y}) \mathrm{d} S_{y}
\end{gathered}
$$

so that, adding the primary BIE (4) and the previous identity, one gets the following alternative primary BIE in terms of the scattered wave $p^{I}$ and the total pressure $p^{T}$ :

$$
p(\boldsymbol{x})+\int_{\Gamma} p^{T}(\boldsymbol{y})\left[G_{, n}(\boldsymbol{x}, \boldsymbol{y})-G_{, n}^{0}(\boldsymbol{x}, \boldsymbol{y})\right] \mathrm{d} S_{y}+\int_{\Gamma}\left[p^{T}(\boldsymbol{y})-p^{T}(\boldsymbol{x})\right] G_{, n}^{0}(\boldsymbol{x}, \boldsymbol{y}) \mathrm{d} S_{y}=0
$$

We elect to use the above alternative BIE instead of (4) for the purpose of material differentiation because the derivation turns out to be simpler due to the absence of $p_{, n}^{I}$ in (56). The differentiation formula (9) is now applied to (56), giving after some manipulations sketched in Appendix $\mathrm{C}$ the sought derivative BIE as follows:

$$
\begin{aligned}
\stackrel{\star}{p}(\boldsymbol{x})+\int_{\Gamma} \stackrel{\star}{p}^{T}(\boldsymbol{y})\left[G_{, n}(\boldsymbol{x}, \boldsymbol{y})-G_{, n}^{0}(\boldsymbol{x}, \boldsymbol{y})\right] \mathrm{d} S_{y}+\int_{\Gamma}\left[\stackrel{\star}{p}^{T}(\boldsymbol{y})-\stackrel{\star}{p}^{T}(\boldsymbol{x})\right] G_{, n}^{0}(\boldsymbol{x}, \boldsymbol{y}) \mathrm{d} S_{y} \\
\quad=\int_{\Gamma}\left\{n_{i}(\boldsymbol{y}) k^{2} G(\boldsymbol{x}, \boldsymbol{y}) p^{T}(\boldsymbol{y})-D_{i j} p^{T}(\boldsymbol{y}) G_{, j}(\boldsymbol{x}, \boldsymbol{y})\right\}\left[\left(\theta n_{i}\right)(\boldsymbol{y})-\left(\theta n_{i}\right)(\boldsymbol{x})\right] \mathrm{d} S_{y}
\end{aligned}
$$


where $D_{r s}$ is the tangential differential operator (66). The validity of the calculation leading to the above result is a by-product of the weakly singular character of the acoustic BIE (56). The derivative BIE (57) is valid for a smooth surface only, without edges or corners (but a similar derivative BIE can be formulated for the more general case of piecewise smooth surfaces). All integrands in eqn. (57) are weakly singular due to the regularizing effect of the factors $\stackrel{\star}{p}(\boldsymbol{y})-\stackrel{\star}{p}(\boldsymbol{x}), \theta(\boldsymbol{y}) n_{s}(\boldsymbol{y})-\theta(\boldsymbol{x}) n_{s}(\boldsymbol{x})$. The right-hand side is expressed in terms of the primary state and in a reasonably compact manner; it shows linear dependence with respect to $\theta$. Note that one has $\stackrel{\star}{p}^{T}=\stackrel{\star}{p}+\stackrel{\star}{p}=\stackrel{\star}{p}-p_{n}^{I} \theta$

Then the required steps for evaluating the derivative (54) in any given geometrical transformation (5) using the direct differentiation approach are as follows:

1. Solve the primary BIE (4) for $p_{\Gamma}$ (or, equivalently, (56) for the unknown $p_{\Gamma}^{T}$.

2. Solve the derivative BIE (57) for the unknown $\stackrel{\star}{p}_{\Gamma}^{T}$.

3. Compute $p_{\Gamma}, p_{\Gamma}^{\star}$ on the measurement surface $C$ using (56), (57) as representation formulas.

4. Compute $\stackrel{\star}{\mathcal{J}}(54)$.

Performing the previous process for all possible $\left.\theta\right|_{\Gamma}$ yields the infinite-dimensional gradient of $\mathcal{J}$. In practical applications, one considers domain changes described by a finite number $D$ of parameters. Then the finitedimensional gradient of $\mathcal{J}$ is computed using $D$ distinct velocities $\theta$ adequately constructed and through the solution of (57) for the $D$ right-hand sides associated to the $D$ velocities.

The right-hand side of (57) is explicit once the primary BIE (56) is solved. The successive solution of (57) for various $\theta$ s may look at first sight to be a heavy computational task. However, the same integral operator governs $p$ in (56) and $\stackrel{\star}{p}$ in (57). The computation of the solution $\stackrel{\star}{p}$ to (57) for a given $\theta$ reuses the already build and factored matrix operator and needs only one right-hand side set-up followed by one backsubstitution.

Extension to elastodynamics. Following the same lines as above, the governing BIE for elastic scattering by a traction-free cavity can be formulated as:

$$
u_{k}(\boldsymbol{x})+\int_{\Gamma} u_{i}^{T}(\boldsymbol{y})\left[\sum_{i j}^{k}(\boldsymbol{x}, \boldsymbol{y} ; \omega)-\Sigma_{i j}^{k}(\boldsymbol{x}, \boldsymbol{y})\right] n_{j}(\boldsymbol{y}) \mathrm{d} S_{y}+\int_{\Gamma}\left[u_{i}^{T}(\boldsymbol{y})-u_{i}^{T}(\boldsymbol{x})\right] \Sigma_{i j}^{k}(\boldsymbol{x}, \boldsymbol{y}) n_{j}(\boldsymbol{y}) \mathrm{d} S_{y}=0
$$

where $U^{k}(\boldsymbol{x}, \boldsymbol{y} ; \omega), \Sigma^{k}(\boldsymbol{x}, \boldsymbol{y} ; \omega)$ denote the fundamental elastodynamic displacement and stress fields at $\boldsymbol{y}$ created in the infinite space by a unit time-harmonic point force applied at $\boldsymbol{x}$ along the $k$-direction, $U^{k}(\boldsymbol{x}, \boldsymbol{y}), \Sigma^{k}(\boldsymbol{x}, \boldsymbol{y})$ being the corresponding static fundamental solution. It is then left to the reader to show that, similarly to the acoustic case, the following derivative BIE holds:

$$
\begin{aligned}
& \stackrel{\star}{u}_{k}(\boldsymbol{x})+\int_{\Gamma} \stackrel{\star}{u}_{i}^{T}(\boldsymbol{y})\left[\sum_{i j}^{k}(\boldsymbol{x}, \boldsymbol{y} ; \omega)-\Sigma_{i j}^{k}(\boldsymbol{x}, \boldsymbol{y})\right] n_{j}(\boldsymbol{y}) \mathrm{d} S_{y} \\
& +\int_{\Gamma}\left[{ }^{\star_{i}^{T}}(\boldsymbol{y})-\stackrel{\star \star}{u}_{i}^{T}(\boldsymbol{x})\right] \sum_{i j}^{k}(\boldsymbol{x}, \boldsymbol{y}) n_{j}(\boldsymbol{y}) \mathrm{d} S_{y} \\
& =\int_{\Gamma} \rho \omega^{2} U_{i}^{k}(\boldsymbol{x}, \boldsymbol{y}) u_{i}^{T}(\boldsymbol{y})\left[\theta(\boldsymbol{y})-n_{m}(\boldsymbol{y}) n_{m}(\boldsymbol{x}) \theta(\boldsymbol{x})\right] \mathrm{d} S_{y} \\
& -\int_{\Gamma} D_{j m} u_{i}^{T}(\boldsymbol{y})\left[\left(\theta n_{m}\right)(\boldsymbol{y})-\left(\theta n_{m}\right)(\boldsymbol{x})\right] \sum_{i j}^{k}(\boldsymbol{x}, \boldsymbol{y})(\boldsymbol{x}, \boldsymbol{y}) \mathrm{d} S_{y}
\end{aligned}
$$




\section{Concluding comments and perspectives}

The approach presented here on particular classes of shape identification problems can be extended to many other situations, involving bounded bodies, other kinds of cost functions, other physical contexts like heat conduction,... It is essentially a numerical tool, in that it allows an optimal use of classical unconstrained minimization methods using gradient evaluations, applied to the physical model and data at hand. On the other side, it provides no insight on the fundamental characteristics of the identification problem, such as existence or uniqueness of the solution for the available data.

The numerical results presented here show the efficiency of the adjoint problem approach for the computation of functional gradients. The very good results obtained on cases using a moderate number of design parameters validate the good performance of the basic components of the inversion strategy. When a more complex descriptions of the unknown surface, allowing in principle the recovery of more general shapes, is used, results deteriorate and ill-posedness manifests itself in the form of highly oscillating reconstructed surfaces. Hence the inversion methodology should ultimately include a regularization [24] of the inverse problem, by means of a stabilizing positive functional $P(\Gamma)$, so that the unknown surface $\Gamma$ is searched as a minimizer of $R(\Gamma, \alpha)=\mathcal{J}(\Gamma)+\alpha P(\Gamma)(0<\alpha \ll 1)$ instead of $\mathcal{J}(\Gamma)$ alone. A suggestion for the functional $P(\Gamma)$ is:

$$
P(\Gamma)=\frac{1}{2} \int_{\Gamma}\left(\operatorname{div}_{S} \boldsymbol{n}\right)^{2} \mathrm{~d} S+\beta \int_{L}\left(1-\boldsymbol{n}^{+} . \boldsymbol{n}^{-}\right) \mathrm{d} s
$$

where $L$ denotes the set of all edges on $\Gamma$ (including element edges), $\boldsymbol{n}^{+}, \boldsymbol{n}^{-}$are the unit normals adjacent to an edge and $\beta$ is an adjustable coefficient which ensures the dimensional consistency of $P(\Gamma)$. The first integral term allows the penalization of high curvatures, which may affect even the continuous reconstructed shape as a result of data uncertainties, while the second is more specifically intended to damp numerical oscillations of the BE-discretized surface: the jump of unit normals between elements is expected to contribute notably to the unwanted oscillations.

To the author' best knowledge, the practically important issue of finding parametrized representations allowing general shapes for 3D surfaces while keeping the number of design parameters as low as possible, and also being preferably designed so as to avoid the appearance of oscillatory shapes, is not yet well understood.

\section{References}

[1] Aithal, R., Saigal, S. Shape sensitivity analysis in thermal problems using BEM. Engng. Anal. with Bound. Elem., 15, 115-120 (1995).

[2] Barone, M. R., YAng, R. J. A boundary element approach for recovery of shape sensitivities in threedimensional elastic solids. Comp. Meth. in Appl. Mech. Engng., 74, 69-82 (1989).

[3] Bonnet, M. Regularized boundary integral equations for three-dimensional bounded or unbounded elastic bodies containing curved cracks of arbitrary shape under dynamic loading. In N. G. Zamani 
C.A. Brebbia (ed.), Boundary element techniques: applications in engineering, pp. 171-189. Comp. Mech. Publ., Southampton, Southampton (1989).

[4] Bonnet, M. A numerical approach for shape identification problems using BIE and shape differentiation. In F. Paris C.A. Brebbia, J. Dominguez (ed.), Boundary Elements XIV (vol. 2: stress analysis, computational aspects), pp. 541-553. Comp. Mech. Publ., Southampton (1992).

[5] Bonnet, M. Shape identification using acoustic measurements: a numerical investigation using BIE and shape differentiation. In H. D. Bui M. Tanaka (ed.), Inverse problems in engineering mechanics, pp. 191-200. Springer-Verlag (1993). IUTAM Symposium, 11-15 Mai 1992, Tokyo, Japon.

[6] Bonnet, M. Regularized BIE formulations for first- and second-order shape sensitivity of elastic fields. Computers and Structures, 56, 799-811 (1995). (Invited paper, special issue, S. Saigal, guest editor).

[7] Bonnet, M., Bui, H. D. On some inverse problems for determining volumic defects by electric current using BIE approaches: an overview. In Proceedings of the 6th National Japanese Conf. on Boundary Elements Methods (Tokyo, Japan), pp. 179-198 (Dec. 1989).

[8] Bonnet, M., Bui, H. D. Regularization of the displacement and traction BIE for 3D elastodynamics using indirect methods. In J. H. Kane, G. Maier, N. Tosaka, S. N. Atluri (eds.), Advances in Boundary Element Techniques, pp. 1-29. Springer-Verlag (1993).

[9] Bonnet, M., Bui, H. D., Maigre, H., Planchard, J. Identification of heat conduction coefficient: application to nondestructive testing. In H. D. Bui M. Tanaka (ed.), Inverse problems in engineering mechanics, pp. 475-488. Springer-Verlag (1993). IUTAM Symposium, 11-15 Mai 1992, Tokyo, Japon.

[10] Burczyński, T. Boundary element method in stochastic shape design sensitivity analysis and identification of uncertain elastic solids. Engng. Anal. with Bound. Elem., 15, 151-160 (1995).

[11] Choi, J. O., KwaK, B. M. Boundary Integral Equation Method for Shape Optimization of Elastic Structures. Int. J. Num. Meth. in Eng., 26, 1579-1595 (1988).

[12] Colton, D. The inverse scattering problem for time-harmonic acoustic waves. SIAM Review, 26, 323-350 (1984).

[13] Colton, D., Kress, R. Integral Equation Method in Scattering Theory. John Wiley and sons (1983).

[14] Fletcher, R. Practical Methods of Optimization. John Wiley and sons (1987).

[15] Haug, E. J., Choi, K. K., Komkov, V. Design Sensitivity Analysis of Structural Systems. Academic Press (1986).

[16] Kassab, A., Moslehy, F. A., Daryapurkar, A. B. Nondestructive evaluation of cavities by an inverse elastostatics boundary element method. Engng. Anal. with Bound. Elem., 13, 45-55 (1994). 
[17] Kress, R. Integral equation methods in inverse obstacle scattering. Engng. Anal. with Bound. Elem., 15, 171-180 (1995).

[18] Mellings, S. C., Aliabadi, M. H. Three-dimensional flaw identification using sensitivity analysis. In C. A. Brebbia (ed.), Boundary Element Method XVI., pp. 149-156. Comp. Mech. Publ., Southampton (1994).

[19] Meric, R. A. Differential and integral sensitivity formulations and shape optimization by BEM. Engng. Anal. with Bound. Elem., 15, 181-188 (1995).

[20] Nishimura, N, Kobayashi, S. A boundary integral equation method for an inverse problem related to crack detection. Int. J. Num. Meth. in Eng., 32, 1371-1387 (1991).

[21] Nishimura, N, Kobayashi, S. Determination of cracks having arbitrary shape with boundary integral method. Engng. Anal. with Bound. Elem., 15, 189-197 (1995).

[22] Petryk, H., Mróz, Z. Time derivatives of integrals and functionals defined on varying volume and surface domains. Arch. Mech., 38, 694-724 (1986).

[23] Press, W. H., Flannery, B. P., Teukolsky, S. A., Vetterling, W. T. Numerical recipes: the art of scientific computing (second edition). Cambridge press (1992).

[24] Tikhonov, A. N., Arsenin, V. Y. Solutions to ill-posed problems. Winston-Wiley, New York (1977).

[25] Zhang, Q., MukherJee, S. Second-order design sensitivity analysis for linear elastic problems by the derivative boundary element method. Comp. Meth. in Appl. Mech. Engng., 86, 321-335 (1991). 


\section{A Tangential differential operators and integration by parts.}

Tangential differential operators. Let $S$ be a twice continuously differentiable closed $C^{2}$ surface, of unit normal $\boldsymbol{n}$ (open surfaces can be considered as well, see e.g. [8]). Consider a scalar function $u(\boldsymbol{y}), \boldsymbol{y} \in S$, which may be undefined outside $S$ (e.g. $u=n_{i}$ or $u=\theta$ ). In this case, the cartesian derivatives $u_{, i}$ are generally meaningless, and one has to introduce tangential differential operators. The domain of definition of $u$ is extended in a neighbourhood $V$ of $S$ by introducing a continuation $\hat{u}$ of $u$ outside $S$ defined as: $\forall(\boldsymbol{y} \in V), \hat{u}(\boldsymbol{y})=u(P(\boldsymbol{y}))$, where $P(\boldsymbol{y})$ is the orthogonal projection of $\boldsymbol{y}$ onto $S$. Clearly the restriction of $\hat{u}$ to $S$ is equal to $u$. Moreover the normal derivative of $\hat{u}$ is equal to zero, i.e. the vector $\nabla \hat{u}$ is tangent to $S$; therefore it may be used to define the tangential gradient $\nabla_{S} u$ of $u$;

$$
\nabla_{S} u=\nabla_{S} \hat{u}=\nabla \hat{u}
$$

If $u$ is an arbitrary scalar function defined in $V$, one has, consistently with (61):

$$
\nabla_{S} u=\nabla u-\boldsymbol{n} u_{, n}=e_{r} \hat{D}_{r} u=e_{r}\left(u_{, r}-n_{r} u_{, n}\right)
$$

which defines the tangential partial derivatives $\hat{D}_{r} u$ (using the notation $(\cdot)_{, n}=\partial / \partial n(\cdot)$ ). The symbol $\left(^{\wedge}\right.$ ) will be omitted, keeping in mind if necessary the extension. Similarly, the surface divergence $\operatorname{div}_{S}$ of a vector or tensor field $\boldsymbol{T}$ is defined as:

$$
\operatorname{div}_{S} \boldsymbol{T}=\operatorname{div} \boldsymbol{T}-\boldsymbol{T}_{, n} \cdot \boldsymbol{n}
$$

so that, when $\boldsymbol{T}$ is a vector $\boldsymbol{u}$ or second-order tensor $\boldsymbol{\sigma}$, one has, referring to a fixed orthonormal frame:

$$
\operatorname{div}_{S} \boldsymbol{u}=D_{j} u_{j} \quad \operatorname{div}_{S} \boldsymbol{\sigma}=D_{j} \sigma_{i j} \boldsymbol{e}_{i}
$$

An interesting consequence of (62) is the following identity for the Laplace operator:

$$
\Delta u=u_{, n n}-2 K u_{, n}+\operatorname{div}_{S}\left(\nabla_{S} u\right)
$$

where $K$, the mean curvature of $S$, is also given by:

$$
2 K=-\operatorname{div}_{S} \boldsymbol{n}
$$

The operator

$$
D_{r s} f=\left(n_{r} f_{, s}-n_{s} f_{, r}\right)
$$

is also introduced. From (62), $D_{r s} f=n_{r} D_{s} f-n_{s} D_{r} f: D_{r s} f$ is a tangential differential operator.

Integration by parts along surfaces. The classical Stokes' identity for a vector field $\boldsymbol{U}$ defined over $\mathrm{V}$ reads:

$$
e_{a b c} \int_{S} n_{a} U_{b, c} \mathrm{~d} S=0
$$

Its application to the special choice $U_{b}=e_{r b s} u$ yields the following integration by parts formula associated to the operator $D_{r s}(66)$, which allows integration by parts on surfaces of combinations of $\boldsymbol{n}$ and ordinary partial derivatives (i.e. without separation of tangential and normal derivatives):

$$
\int_{S} D_{r s} u \mathrm{~d} S=0 \quad \text { for any fixed pair } r, s, \quad r, s=1,2,3
$$


Then the special choice $u=v_{r} n_{s}$ in (68) gives in turn:

$$
\int_{S}\left(2 K \boldsymbol{v} \cdot \boldsymbol{n}+\operatorname{div}_{S} \boldsymbol{v}\right) \mathrm{d} S=0
$$

\section{B Some auxiliary formulas}

The following formulas are given e.g. in [22]:

$$
\begin{aligned}
\stackrel{\star}{\boldsymbol{n}} & =-\boldsymbol{\nabla}_{S} \theta=-\left(D_{r} \theta\right) \boldsymbol{e}_{r} \\
(\boldsymbol{\nabla} u)^{\star} & =\boldsymbol{\nabla} \stackrel{\star}{u}-u_{, n} \boldsymbol{\nabla} \theta-\theta \boldsymbol{\nabla}_{S} u . \nabla_{S} \boldsymbol{n}
\end{aligned}
$$

Their combined application leads to the following formula for the transformation derivative $\left(u_{, n}\right)^{\star}$ :

$$
(u, n)^{\star}=\stackrel{\star}{u, n}-u_{, n} \theta_{, n}-\nabla_{S} u \nabla_{S} \theta
$$

Since the definition of the incident wave $p^{I}$ does not depend on the actual location of $\Gamma$, one has $p_{, \tau}^{I}=0$ i.e. $\stackrel{\star}{p}=\theta p_{, n}^{I}$, so that $(70)$ gives:

$$
\left(p_{, n}^{I}\right)^{\star}=\theta p_{, n n}^{I}-\nabla_{S} p^{I} \cdot \nabla_{S} \theta
$$

Moreover, since $p^{I}$ solves the Helmholtz equation, identity (64) gives:

$$
p_{, n n}^{I}=2 K p_{, n}^{I}-\operatorname{div}_{S}\left(\theta \nabla_{S} p^{I}\right)-k^{2} p^{I}
$$

Equation (14) is then readily established from the last two equations.

\section{Proof of equation (57)}

First, as the fundamental solutions $G(\boldsymbol{x}, \boldsymbol{y}), G^{0}(\boldsymbol{x}, \boldsymbol{y})$ do not depend on $\tau$, one has, in component notation:

$$
\left(G_{, n}\right)^{\star}(\boldsymbol{x}, \boldsymbol{y})=G_{, i j}(\boldsymbol{x}, \boldsymbol{y}) n_{j}(\boldsymbol{y})\left[\left(\theta n_{i}\right)(\boldsymbol{y})-\left(\theta n_{i}\right)(\boldsymbol{x})\right]-D_{j} G(\boldsymbol{x}, \boldsymbol{y}) D_{j} \theta(\boldsymbol{y})
$$

This result uses the fact that $G(\boldsymbol{x}, \boldsymbol{y})=G(\boldsymbol{y}, \boldsymbol{x})$; the derivatives $\left({ }_{, j}, D_{j}\right.$ are taken with respect to field point coordinates $y_{j}$. Upon application of (9), one has:

$$
\begin{aligned}
& \frac{d}{d \tau} \int_{\Gamma} p(\boldsymbol{y})\left[G_{, n}(\boldsymbol{x}, \boldsymbol{y})-G_{, n}^{0}(\boldsymbol{x}, \boldsymbol{y})\right] \mathrm{d} S_{y} \\
& =\int_{\Gamma}{ }_{p}^{\star}(\boldsymbol{y})\left[G_{, n}(\boldsymbol{x}, \boldsymbol{y})-G_{, n}^{0}(\boldsymbol{x}, \boldsymbol{y})\right] \mathrm{d} S_{y} \\
& \quad+\int_{\Gamma} p(\boldsymbol{y})\left[G(\boldsymbol{x}, \boldsymbol{y})-G^{0}(\boldsymbol{x}, \boldsymbol{y})\right]_{, i j} n_{j}(\boldsymbol{y})\left[\left(\theta n_{i}\right)(\boldsymbol{y})-\left(\theta n_{i}\right)(\boldsymbol{x})\right] \mathrm{d} S_{y} \\
& \quad-\int_{\Gamma}\left\{D_{j} G(\boldsymbol{x}, \boldsymbol{y}) D_{j} \theta(\boldsymbol{y})+2 K G_{, n}(\boldsymbol{x}, \boldsymbol{y}) \theta(\boldsymbol{y})\right\} \mathrm{d} S_{y}
\end{aligned}
$$


Next, one can rewrite the last integral above using the $D_{i j}$ operator (66) and the corresponding integration by parts identity (68):

$$
\begin{aligned}
& \int_{\Gamma} p(\boldsymbol{y})\left[G(\boldsymbol{x}, \boldsymbol{y})-G^{0}(\boldsymbol{x}, \boldsymbol{y})\right]_{, i j} n_{j}(\boldsymbol{y})\left[\left(\theta n_{i}\right)(\boldsymbol{y})-\left(\theta n_{i}\right)(\boldsymbol{x})\right] \mathrm{d} S_{y} \\
& =\int_{\Gamma} p(\boldsymbol{y})\left\{D_{j i}\left[G(\boldsymbol{x}, \boldsymbol{y})-G^{0}(\boldsymbol{x}, \boldsymbol{y})\right]_{, j}+n_{i}(\boldsymbol{y})\left[G(\boldsymbol{x}, \boldsymbol{y})-G^{0}(\boldsymbol{x}, \boldsymbol{y})\right]_{, j}\right\}\left[\left(\theta n_{i}\right)(\boldsymbol{y})-\left(\theta n_{i}\right)(\boldsymbol{x})\right] \mathrm{d} S_{y} \\
& =\int_{\Gamma}\left\{D_{i j} p(\boldsymbol{y})\left[G(\boldsymbol{x}, \boldsymbol{y})-G^{0}(\boldsymbol{x}, \boldsymbol{y})\right]_{, j}-n_{i}(\boldsymbol{y}) p(\boldsymbol{y}) k^{2} G(\boldsymbol{x}, \boldsymbol{y})\right\}\left[\left(\theta n_{i}\right)(\boldsymbol{y})-\left(\theta n_{i}\right)(\boldsymbol{x})\right] \mathrm{d} S_{y} \\
& \quad+\int_{\Gamma} p(\boldsymbol{y})\left[G(\boldsymbol{x}, \boldsymbol{y})-G^{0}(\boldsymbol{x}, \boldsymbol{y})\right]_{, j} D_{i j}\left(\theta n_{i}\right)(\boldsymbol{y}) \mathrm{d} S_{y} \\
& =\int_{\Gamma}\left\{D_{i j} p(\boldsymbol{y})\left[G(\boldsymbol{x}, \boldsymbol{y})-G^{0}(\boldsymbol{x}, \boldsymbol{y})\right]_{, j}-n_{i}(\boldsymbol{y}) p(\boldsymbol{y}) k^{2} G(\boldsymbol{x}, \boldsymbol{y})\right\}\left[\left(\theta n_{i}\right)(\boldsymbol{y})-\left(\theta n_{i}\right)(\boldsymbol{x})\right] \mathrm{d} S_{y} \\
& \quad-\int_{\Gamma} p(\boldsymbol{y})\left[G(\boldsymbol{x}, \boldsymbol{y})-G^{0}(\boldsymbol{x}, \boldsymbol{y})\right]_{, j}\left[D_{j} \theta(\boldsymbol{y})+2 K n_{j}(\boldsymbol{y}) \mathrm{d} S_{y}\right.
\end{aligned}
$$

where the identities $G_{, j j}+k^{2} G=-\delta(\boldsymbol{y}-\boldsymbol{x})$ and $G_{, j j}^{0}=-\delta(\boldsymbol{y}-\boldsymbol{x})$ were used, together with:

$$
\begin{aligned}
D_{i j}\left(n_{i} \theta(\boldsymbol{y})\right) & =n_{i}\left[n_{i} D_{j} \theta+\theta D_{j} n_{i}\right]-n_{j}\left[n_{i} D_{i} \theta+\theta D_{i} n_{i}\right] \\
& =D_{j} \theta-2 K n_{j} \theta
\end{aligned}
$$

Summing up, one has:

$$
\begin{aligned}
& \frac{d}{d \tau} \int_{\Gamma} p(\boldsymbol{y})\left[G_{, n}(\boldsymbol{x}, \boldsymbol{y})-G_{, n}^{0}(\boldsymbol{x}, \boldsymbol{y})\right] \mathrm{d} S_{y} \\
& =\int_{\Gamma}{ }_{p}^{\star}(\boldsymbol{y})\left[G_{, n}(\boldsymbol{x}, \boldsymbol{y})-G_{, n}^{0}(\boldsymbol{x}, \boldsymbol{y})\right] \mathrm{d} S_{y} \\
& \quad+\int_{\Gamma}\left\{D_{i j} p(\boldsymbol{y})\left[G(\boldsymbol{x}, \boldsymbol{y})-G^{0}(\boldsymbol{x}, \boldsymbol{y})\right]_{, j}-n_{i}(\boldsymbol{y}) p(\boldsymbol{y}) k^{2} G(\boldsymbol{x}, \boldsymbol{y})\right\}\left[\left(\theta n_{i}\right)(\boldsymbol{y})-\left(\theta n_{i}\right)(\boldsymbol{x})\right] \mathrm{d} S_{y}
\end{aligned}
$$

A similar calculation also shows that:

$$
\begin{aligned}
& \frac{d}{d \tau} \int_{\Gamma}[p(\boldsymbol{y})-p(\boldsymbol{y})] G^{0}(\boldsymbol{x}, \boldsymbol{y}) \mathrm{d} S_{y} \\
& =\int_{\Gamma}[p(\boldsymbol{y})-\stackrel{\star}{p}(\boldsymbol{x})] G_{, n}^{0}(\boldsymbol{x}, \boldsymbol{y}) \mathrm{d} S_{y} \\
& \left.\quad+\int_{\Gamma} D_{i j} p(\boldsymbol{y}) G^{0}(\boldsymbol{x}, \boldsymbol{y})\right]_{, j}\left[\left(\theta n_{i}\right)(\boldsymbol{y})-\left(\theta n_{i}\right)(\boldsymbol{x})\right] \mathrm{d} S_{y}
\end{aligned}
$$

The result (57) then follows readily from the last two equations. 


\section{List of Tables}

1 'True' and initial $\left(d_{1}, \ldots, d_{9}\right)$ and wavenumber values: examples 1 to $6 . \ldots \ldots$

2 Results for example $1 . \ldots \ldots \ldots \ldots \ldots \ldots$

3 Results for example $2 \ldots \ldots \ldots \ldots \ldots \ldots \ldots \ldots$

4 Results for example $3 . \ldots \ldots \ldots \ldots \ldots \ldots \ldots$

$5 \quad$ Results for example $4 . \ldots \ldots \ldots \ldots \ldots \ldots \ldots$

6 Results for example $5 . \ldots \ldots \ldots \ldots \ldots$

$7 \quad$ Results for example $6 \ldots \ldots \ldots \ldots \ldots \ldots \ldots$

8 Converged values of $e_{V}, e_{A}, e_{I}, \mathcal{J}_{\text {final }} / \mathcal{J}_{\text {initial }}$ for example 7: comparison between descriptions of $\Gamma$ using 9 geometrical parameters and 222 nodal coordinates. . . . . . . . . . . . . . . 32 


\begin{tabular}{|l|c||ccc|ccc|ccc|}
\hline Example no. & & $x_{G}$ & $y_{G}$ & $z_{G}$ & $\phi$ & $\theta$ & $\psi$ & $a$ & $b$ & $c$ \\
\hline \hline \multirow{2}{*}{$\begin{array}{l}1 \\
(k=1)\end{array}$} & True & 1.0 & 1.0 & 0.5 & 0.4 & 0.9 & 0.6 & 1.0 & 2.0 & 1.0 \\
\cline { 2 - 10 } & Initial & 1.0 & 1.0 & 0.5 & 0.4 & 0.9 & 0.6 & 1.2 & 2.4 & 1.2 \\
\hline \hline \multirow{2}{*}{$(k=.3)$} & True & 1.0 & 1.0 & 0.5 & 0.4 & 0.9 & 0.6 & 1.0 & 2.0 & 1.0 \\
\cline { 2 - 9 } & Initial & 0.0 & 0.0 & 0.0 & 0.0 & 0.0 & 0.0 & 4.0 & 4.0 & 4.0 \\
\hline \hline \multirow{2}{*}{$\begin{array}{l}3,4,5,6 \\
(k=.5)\end{array}$} & True & 1.0 & 0.0 & -2.0 & 0.4 & 0.9 & 0.6 & 1.0 & 3.0 & 1.0 \\
\cline { 2 - 10 } & Initial & 0.0 & 0.0 & 0.0 & 0.0 & 0.0 & 0.0 & 1.5 & 1.5 & 1.5 \\
\hline
\end{tabular}

Table 1: 'True' and initial $\left(d_{1}, \ldots, d_{9}\right)$ and wavenumber values: examples 1 to 6 . 


\begin{tabular}{|c|c|l|l|l|l|}
\hline \multicolumn{2}{|c|}{ Example 1} & $\epsilon=0$. & $\epsilon=10^{-3}$ & $\epsilon=10^{-2}$ & $\epsilon=10^{-1}$ \\
\hline \hline \multirow{3}{*}{$p_{3}^{I}$} & $J_{\text {final }} / J_{0}$ & $1.4010^{-8}$ & $4.7310^{-6}$ & $4.8210^{-4}$ & $4.3810^{-2}$ \\
\cline { 2 - 6 } & $e_{V}$ & $2.9110^{-6}$ & $6.2110^{-5}$ & $6.4410^{-4}$ & $6.2310^{-3}$ \\
\cline { 2 - 6 } & $e_{A}$ & $6.7410^{-6}$ & $1.1110^{-4}$ & $1.1610^{-3}$ & $1.1410^{-2}$ \\
\cline { 2 - 6 } & $e_{I}$ & $1.5710^{-5}$ & $3.0810^{-4}$ & $3.1210^{-3}$ & $3.1710^{-2}$ \\
\cline { 2 - 6 } & Evaluations & 68 & 38 & 37 & 37 \\
\hline \hline \multirow{7}{*}{$p_{2}^{I}, p_{3}^{I}$} & $J_{\text {final }} / J_{0}$ & $2.6510^{-9}$ & $6.9710^{-6}$ & $7.0110^{-4}$ & $6.4210^{-2}$ \\
\cline { 2 - 6 } & $e_{V}$ & $4.4610^{-7}$ & $1.1010^{-4}$ & $1.1110^{-3}$ & $1.1110^{-2}$ \\
\cline { 2 - 6 } & $e_{A}$ & $3.7110^{-7}$ & $7.7710^{-5}$ & $7.8110^{-4}$ & $7.8310^{-3}$ \\
\cline { 2 - 6 } & $e_{I}$ & $4.5310^{-6}$ & $1.3110^{-4}$ & $1.2910^{-3}$ & $1.2910^{-2}$ \\
\cline { 2 - 6 } & Evaluations & 50 & 23 & 38 & 28 \\
\hline \hline$p_{1}^{I}, p_{2}^{I}, p_{3}^{I}$ & $J_{\text {final }} / J_{0}$ & $9.0710^{-13}$ & $1.1810^{-5}$ & $1.1710^{-3}$ & $9.9210^{-2}$ \\
\cline { 2 - 6 } & $e_{V}$ & $1.2110^{-8}$ & $5.7310^{-5}$ & $5.7310^{-4}$ & $5.6210^{-3}$ \\
\cline { 2 - 6 } & $e_{A}$ & $1.6810^{-9}$ & $4.2210^{-5}$ & $4.2210^{-4}$ & $4.3810^{-3}$ \\
\cline { 2 - 6 } & $e_{I}$ & $1.9410^{-8}$ & $4.1610^{-5}$ & $4.1610^{-4}$ & $4.8410^{-3}$ \\
\cline { 2 - 6 } & Evaluations & 42 & 19 & 18 & 72 \\
\hline
\end{tabular}

Table 2: Results for example 1. 


\begin{tabular}{|c|c|l|l|l|l|}
\hline \multicolumn{2}{|c|}{ Example 2 } & $\epsilon=0$. & $\epsilon=10^{-3}$ & $\epsilon=10^{-2}$ & $\epsilon=10^{-1}$ \\
\hline \hline \multirow{3}{*}{$p_{3}^{I}$} & $J_{\text {final }} / J_{0}$ & $1.4810^{-11}$ & $9.0110^{-10}$ & $9.2310^{-8}$ & $9.3810^{-6}$ \\
\cline { 2 - 6 } & $e_{V}$ & $1.0410^{-5}$ & $1.1710^{-5}$ & $3.8610^{-4}$ & $3.2010^{-3}$ \\
\cline { 2 - 6 } & $e_{A}$ & $3.4110^{-5}$ & $4.4610^{-4}$ & $3.2310^{-4}$ & $7.0310^{-4}$ \\
\cline { 2 - 6 } & $e_{I}$ & $2.3310^{-4}$ & $1.2110^{-3}$ & $5.6410^{-3}$ & $2.2810^{-1}$ \\
\cline { 2 - 6 } & Evaluations & 139 & 122 & 129 & 67 \\
\hline \hline \multirow{7}{*}{$p_{2}^{I}, p_{3}^{I}$} & $J_{\text {final }} / J_{0}$ & $3.3310^{-11}$ & $8.5910^{-10}$ & $8.8710^{-8}$ & $8.3010^{-6}$ \\
\cline { 2 - 6 } & $e_{V}$ & $7.2910^{-5}$ & $3.4510^{-5}$ & $2.3110^{-4}$ & $2.9110^{-4}$ \\
\cline { 2 - 6 } & $e_{A}$ & $1.1910^{-4}$ & $4.1810^{-5}$ & $4.4510^{-4}$ & $2.3610^{-3}$ \\
\cline { 2 - 6 } & $e_{I}$ & $2.3410^{-4}$ & $1.6710^{-4}$ & $3.1510^{-3}$ & $2.6110^{-2}$ \\
\cline { 2 - 6 } & Evaluations & 103 & 112 & 87 & 101 \\
\hline \hline \multirow{7}{*}{$p_{1}^{I}, p_{2}^{I}, p_{3}^{I}$} & $J_{\text {final }} / J_{0}$ & $2.6110^{-11}$ & $9.1010^{-10}$ & $8.8710^{-8}$ & $8.8810^{-6}$ \\
\cline { 2 - 6 } & $e_{V}$ & $1.9310^{-6}$ & $2.4810^{-5}$ & $2.3110^{-4}$ & $2.2610^{-3}$ \\
\cline { 2 - 6 } & $e_{A}$ & $8.3310^{-7}$ & $4.5210^{-5}$ & $4.4510^{-4}$ & $4.3610^{-3}$ \\
\cline { 2 - 6 } & $e_{I}$ & $5.1910^{-5}$ & $3.0710^{-4}$ & $3.1510^{-3}$ & $3.1310^{-2}$ \\
\cline { 2 - 6 } & Evaluations & 78 & 88 & 87 & 80 \\
\hline
\end{tabular}

Table 3: Results for example 2. 


\begin{tabular}{|c|c|l|l|l|l|}
\hline \multicolumn{2}{|c|}{ Example 3 } & $\epsilon=0$. & $\epsilon=10^{-3}$ & $\epsilon=10^{-2}$ & $\epsilon=10^{-1}$ \\
\hline \hline \multirow{3}{*}{$p_{3}^{I}$} & $J_{\text {final }} / J_{0}$ & $1.8710^{-8}$ & $1.5510^{-7}$ & $1.2710^{-5}$ & $1.2510^{-3}$ \\
\cline { 2 - 6 } & $e_{V}$ & $1.3710^{-5}$ & $4.9810^{-5}$ & $5.8310^{-4}$ & $5.4310^{-3}$ \\
\cline { 2 - 6 } & $e_{A}$ & $9.0910^{-5}$ & $2.6710^{-6}$ & $7.2910^{-4}$ & $7.0110^{-3}$ \\
\cline { 2 - 6 } & $e_{I}$ & $3.6610^{-4}$ & $1.3410^{-4}$ & $1.9710^{-3}$ & $2.1410^{-2}$ \\
\cline { 2 - 6 } & Evaluations & 75 & 74 & 81 & 76 \\
\hline \hline \multirow{3}{*}{$p_{2}^{I}, p_{3}^{I}$} & $J_{\text {final }} / J_{0}$ & $1.4710^{-7}$ & $4.1810^{-7}$ & $2.4910^{-5}$ & $2.4510^{-3}$ \\
\cline { 2 - 6 } & $e_{V}$ & $2.1710^{-5}$ & $9.0510^{-5}$ & $1.0610^{-3}$ & $1.1110^{-2}$ \\
\cline { 2 - 6 } & $e_{A}$ & $6.0210^{-5}$ & $1.9710^{-4}$ & $1.4210^{-3}$ & $1.3510^{-2}$ \\
\cline { 2 - 6 } & $e_{I}$ & $1.6910^{-4}$ & $3.6310^{-4}$ & $2.1510^{-3}$ & $1.9610^{-2}$ \\
\cline { 2 - 6 } & Evaluations & 53 & 52 & 51 & 75 \\
\hline \hline$p_{1}^{I}, p_{2}^{I}, p_{3}^{I}$ & $J_{\text {final }} / J_{0}$ & $1.5910^{-7}$ & $4.1910^{-7}$ & $2.4110^{-5}$ & $2.4210^{-3}$ \\
\cline { 2 - 6 } & $e_{V}$ & $8.5010^{-7}$ & $1.5610^{-4}$ & $1.5610^{-3}$ & $1.5210^{-2}$ \\
\cline { 2 - 6 } & $e_{A}$ & $5.9810^{-6}$ & $1.1710^{-4}$ & $1.1110^{-3}$ & $1.0610^{-2}$ \\
\cline { 2 - 6 } & $e_{I}$ & $7.4610^{-5}$ & $1.6710^{-4}$ & $1.0110^{-3}$ & $8.8010^{-3}$ \\
\cline { 2 - 6 } & Evaluations & 37 & 37 & 35 & 40 \\
\hline
\end{tabular}

Table 4: Results for example 3. 


\begin{tabular}{|c|c|l|l|l|l|}
\hline \multicolumn{2}{|c|}{ Example 4} & $\epsilon=0$. & $\epsilon=10^{-3}$ & $\epsilon=10^{-2}$ & $\epsilon=10^{-1}$ \\
\hline \hline \multirow{3}{*}{$p_{3}^{I}$} & $J_{\text {final }} / J_{0}$ & $3.0110^{-4}$ & $3.0310^{-4}$ & $3.3210^{-4}$ & $2.3410^{-3}$ \\
\cline { 2 - 6 } & $e_{V}$ & $1.7010^{-2}$ & $1.7010^{-2}$ & $1.7510^{-2}$ & $2.2210^{-2}$ \\
\cline { 2 - 6 } & $e_{A}$ & $1.5110^{-1}$ & $1.5110^{-1}$ & $1.5110^{-1}$ & $1.5610^{-1}$ \\
\cline { 2 - 6 } & $e_{I}$ & $5.7410^{-2}$ & $5.7610^{-2}$ & $5.8710^{-2}$ & $7.2410^{-2}$ \\
\cline { 2 - 6 } & Evaluations & 62 & 62 & 63 & 51 \\
\hline \hline \multirow{7}{*}{$p_{2}^{I}, p_{3}^{I}$} & $J_{\text {final }} / J_{0}$ & $4.0910^{-2}$ & $4.1110^{-2}$ & $4.5710^{-2}$ & $3.8810^{-1}$ \\
\cline { 2 - 6 } & $e_{V}$ & $7.9510^{-3}$ & $8.0710^{-3}$ & $9.1510^{-3}$ & $1.9510^{-2}$ \\
\cline { 2 - 6 } & $e_{A}$ & $1.5110^{-1}$ & $1.5110^{-1}$ & $1.5210^{-1}$ & $1.6110^{-1}$ \\
\cline { 2 - 6 } & $e_{I}$ & $5.8410^{-2}$ & $5.8610^{-2}$ & $6.0410^{-2}$ & $7.8510^{-2}$ \\
\cline { 2 - 6 } & Evaluations & 30 & 31 & 41 & 59 \\
\hline \hline$p_{1}^{I}, p_{2}^{I}, p_{3}^{I}$ & $J_{\text {final }} / J_{0}$ & $2.7610^{-3}$ & $2.7710^{-3}$ & $2.8210^{-3}$ & $6.3610^{-3}$ \\
\cline { 2 - 6 } & $e_{V}$ & $1.0010^{-2}$ & $1.0210^{-2}$ & $1.1610^{-2}$ & $2.5410^{-2}$ \\
\cline { 2 - 6 } & $e_{A}$ & $1.3410^{-1}$ & $1.3410^{-1}$ & $1.3510^{-1}$ & $1.4210^{-1}$ \\
\cline { 2 - 6 } & $e_{I}$ & $2.6010^{-2}$ & $2.6110^{-2}$ & $2.6910^{-2}$ & $3.3910^{-2}$ \\
\cline { 2 - 6 } & Evaluations & 41 & 30 & 42 & 60 \\
\hline
\end{tabular}

Table 5: Results for example 4. 


\begin{tabular}{|c|c|l|l|l|l|}
\hline \multicolumn{2}{|c|}{ Example 5 } & $\epsilon=0$. & $\epsilon=10^{-3}$ & $\epsilon=10^{-2}$ & $\epsilon=10^{-1}$ \\
\hline \hline \multirow{3}{*}{$p_{3}^{I}$} & $J_{\text {final }} / J_{0}$ & $1.8410^{-8}$ & $1.4210^{-7}$ & $1.2810^{-5}$ & $1.2510^{-3}$ \\
\cline { 2 - 6 } & $e_{V}$ & $1.6810^{-5}$ & $8.2110^{-5}$ & $1.5510^{-4}$ & $4.8310^{-3}$ \\
\cline { 2 - 6 } & $e_{A}$ & $7.9210^{-5}$ & $1.8810^{-5}$ & $2.7810^{-3}$ & $8.7310^{-3}$ \\
\cline { 2 - 6 } & $e_{I}$ & $3.5810^{-4}$ & $1.4010^{-4}$ & $2.1710^{-3}$ & $2.1510^{-2}$ \\
\cline { 2 - 6 } & Evaluations & 138 & 125 & 81 & 93 \\
\hline \hline \multirow{7}{*}{$p_{2}^{I}, p_{3}^{I}$} & $J_{\text {final }} / J_{0}$ & $1.4710^{-7}$ & $4.1710^{-7}$ & $2.4810^{-5}$ & $2.4310^{-3}$ \\
\cline { 2 - 6 } & $e_{V}$ & $2.8010^{-5}$ & $5.8110^{-5}$ & $7.3710^{-4}$ & $1.0910^{-2}$ \\
\cline { 2 - 6 } & $e_{A}$ & $1.0010^{-4}$ & $3.8810^{-4}$ & $2.9210^{-3}$ & $1.4410^{-2}$ \\
\cline { 2 - 6 } & $e_{I}$ & $1.7610^{-4}$ & $3.8110^{-4}$ & $2.1310^{-3}$ & $1.9510^{-2}$ \\
\cline { 2 - 6 } & Evaluations & 81 & 87 & 55 & 73 \\
\hline \hline$p_{1}^{I}, p_{2}^{I}, p_{3}^{I}$ & $J_{\text {final }} / J_{0}$ & $1.5910^{-7}$ & $4.1810^{-6}$ & $2.4110^{-5}$ & $2.3510^{-3}$ \\
\cline { 2 - 6 } & $e_{V}$ & $8.8710^{-6}$ & $1.6910^{-4}$ & $1.6310^{-3}$ & $1.4010^{-2}$ \\
\cline { 2 - 6 } & $e_{A}$ & $3.9510^{-5}$ & $4.3010^{-5}$ & $8.1310^{-4}$ & $1.3910^{-2}$ \\
\cline { 2 - 6 } & $e_{I}$ & $5.5710^{-5}$ & $1.3510^{-4}$ & $8.9110^{-4}$ & $1.0710^{-2}$ \\
\cline { 2 - 6 } & Evaluations & 71 & 63 & 76 & 60 \\
\hline
\end{tabular}

Table 6: Results for example 5 . 


\begin{tabular}{|c|c|c|c|c|c|}
\hline \multicolumn{2}{|c|}{ Example 6} & $\epsilon=0$ & $\epsilon=10^{-3}$ & $\epsilon=10^{-2}$ & $\epsilon=10^{-1}$ \\
\hline \multirow[t]{6}{*}{$p_{3}^{I}$} & $J_{\text {final }} / J_{0}$ & $4.5610^{-9}$ & $5.1310^{-5}$ & $6.2510^{-5}$ & $1.9810^{-3}$ \\
\hline & $e_{V}$ & $2.3310^{-5}$ & $5.4010^{-3}$ & $5.4510^{-3}$ & $1.2410^{-2}$ \\
\hline & $e_{A}$ & $1.2310^{-3}$ & $1.0610^{-1}$ & $9.7110^{-2}$ & $8.8710^{-2}$ \\
\hline & $e_{I}$ & $2.7210^{-4}$ & $3.1910^{-2}$ & $3.0210^{-2}$ & $4.9110^{-2}$ \\
\hline & $n$ recovered & 722. & 6.27 & 7.19 & 9.45 \\
\hline & Evaluations & 220 & 109 & 119 & 109 \\
\hline \multirow[t]{6}{*}{$p_{2}^{I}, p_{3}^{I}$} & $J_{\text {final }} / J_{0}$ & $2.4010^{-8}$ & $3.6910^{-7}$ & $3.2910^{-5}$ & $3.3010^{-3}$ \\
\hline & $e_{V}$ & $7.2810^{-6}$ & $1.3910^{-4}$ & $1.2910^{-3}$ & $1.2610^{-2}$ \\
\hline & $e_{A}$ & $1.2110^{-3}$ & $1.1210^{-3}$ & $1.9110^{-3}$ & $1.1010^{-2}$ \\
\hline & $e_{I}$ & $4.7010^{-4}$ & $6.1310^{-4}$ & $2.1610^{-3}$ & $1.9810^{-2}$ \\
\hline & $n$ recovered & 1007. & 1025. & 1120. & 1140. \\
\hline & Evaluations & 220 & 240 & 216 & 145 \\
\hline \multirow[t]{6}{*}{$p_{1}^{I}, p_{2}^{I}, p_{3}^{I}$} & $J_{\text {final }} / J_{0}$ & $7.5810^{-8}$ & $4.4610^{-7}$ & $3.3510^{-5}$ & $3.3510^{-3}$ \\
\hline & $e_{V}$ & $2.0610^{-5}$ & $1.7210^{-4}$ & $1.3210^{-3}$ & $1.5010^{-2}$ \\
\hline & $e_{A}$ & $9.3910^{-4}$ & $1.0310^{-3}$ & $1.8810^{-3}$ & $1.0310^{-2}$ \\
\hline & $e_{I}$ & $1.9110^{-4}$ & $2.3910^{-4}$ & $9.3210^{-4}$ & $8.7110^{-2}$ \\
\hline & $n$ recovered & 1035. & 1057. & 1062. & 1045. \\
\hline & Evaluations & 126 & 121 & 123 & 98 \\
\hline
\end{tabular}

Table 7: Results for example 6 . 


\begin{tabular}{|l||c|c|c|c|}
\hline & $e_{V}$ & $e_{A}$ & $e_{I}$ & $\mathcal{J}_{\text {final }} / \mathcal{J}_{\text {initial }}$ \\
\hline \hline 222 unknowns & $3.210^{-4}$ & $7.610^{-4}$ & $2.3410^{-2}$ & $3.410^{-1}$ \\
\hline 9 unknowns & $9.0710^{-13}$ & $1.2110^{-8}$ & $1.6810^{-9}$ & $1.9410^{-8}$ \\
\hline
\end{tabular}

Table 8: Converged values of $e_{V}, e_{A}, e_{I}, \mathcal{J}_{\text {final }} / \mathcal{J}_{\text {initial }}$ for example 7: comparison between descriptions of $\Gamma$ using 9 geometrical parameters and 222 nodal coordinates. 